ARTICLE

DOI: $10.1038 / s 41467-017-02799-0$

\title{
Identification of a Golgi GPI-N-acetylgalactosamine transferase with tandem transmembrane regions in the catalytic domain
}

Tetsuya Hirata1,2,5, Sushil K. Mishra (10 ${ }^{3}$, Shota Nakamura ${ }^{1}$, Kazunobu Saito ${ }^{1}$, Daisuke Motooka1, Yoko Takada², Noriyuki Kanzawa1,2, Yoshiko Murakami ${ }^{1,2}$, Yusuke Maeda ${ }^{1,2}$, Morihisa Fujita ${ }^{4}$, Yoshiki Yamaguchi ${ }^{3}$ \&

Taroh Kinoshita (1) 1,2

Many eukaryotic proteins are anchored to the cell surface via the glycolipid glycosylphosphatidylinositol (GPI). Mammalian GPIs have a conserved core but exhibit diverse $\mathrm{N}$-acetylgalactosamine (GalNAc) modifications, which are added via a yet unresolved process. Here we identify the Golgi-resident GPI-GaINAc transferase PGAP4 and show by mass spectrometry that PGAP4 knockout cells lose GPI-GalNAc structures. Furthermore, we demonstrate that PGAP4, in contrast to known Golgi glycosyltransferases, is not a single-pass membrane protein but contains three transmembrane domains, including a tandem transmembrane domain insertion into its glycosyltransferase-A fold as indicated by comparative modeling. Mutational analysis reveals a catalytic site, a DXD-like motif for UDP-GalNAc donor binding, and several residues potentially involved in acceptor binding. We suggest that a juxtamembrane region of PGAP4 accommodates various GPI-anchored proteins, presenting their acceptor residue toward the catalytic center. In summary, we present insights into the structure of PGAP4 and elucidate the initial step of GPI-GalNAc biosynthesis.

\footnotetext{
${ }^{1}$ Research Institute for Microbial Diseases, Osaka University, Suita, Osaka 565-0871, Japan. ${ }^{2}$ WPI Immunology Frontier Research Center, Osaka University, Suita, Osaka 565-0871, Japan. ${ }^{3}$ Structural Glycobiology Team, Systems Glycobiology Research Group, RIKEN Global Research Cluster, Wako, Saitama $351-$ 0198, Japan. ${ }^{4}$ Key Laboratory of Carbohydrate Chemistry and Biotechnology, Ministry of Education, School of Biotechnology, Jiangnan University, Wuxi, Jiangsu 214122, China. ${ }^{5}$ Present address: National Institute for Physiological Sciences, National Institutes of Natural Sciences, Okazaki, Aichi 444-8787, Japan. Correspondence and requests for materials should be addressed to T.K. (email: tkinoshi@biken.osaka-u.ac.jp)
} 
G

lycosylphosphatidylinositol (GPI) anchoring is a posttranslational modification of proteins by a glycolipid, GPI. In mammalian cells, $>150$ proteins are modified by GPI for anchoring to the cell surface ${ }^{1}$. The common structure of GPI among organisms is EtNP-6Man $\alpha 1-2 \mathrm{Man} \alpha 1-6 \mathrm{Man} \alpha 1-4 \mathrm{GlcN} \alpha 1-$ 6myo-inositol-phospholipid (where EtNP, Man, and GlcN are ethanolamine phosphate, mannose, and glucosamine, respectively). The 2-position of the first Man linked to GlcN is modified by EtNP in mammalian cells (Fig. 1a). The biosynthesis of GPI occurs in the endoplasmic reticulum (ER) followed by attachment of the GPI to proteins to generate immature GPI-anchored proteins (GPI-APs). The immature GPI-APs then undergo lipid and glycan remodeling. Post-GPI attachment to proteins 1 (PGAP1) removes the acyl chain linked to the 2-position of inositol and then PGAP5 removes EtNP from the second Man in the ER before the GPI-APs exit from the $\mathrm{ER}^{2-4}$. In the Golgi, GPI-APs undergo fatty acid remodeling where PGAP3 removes an $s n$-2-linked unsaturated fatty acid and PGAP2 is involved in reacylation with stearic acid, a saturated fatty acid ${ }^{5,6}$. Fatty acid remodeling is crucial for ensuring that GPI-APs associate with
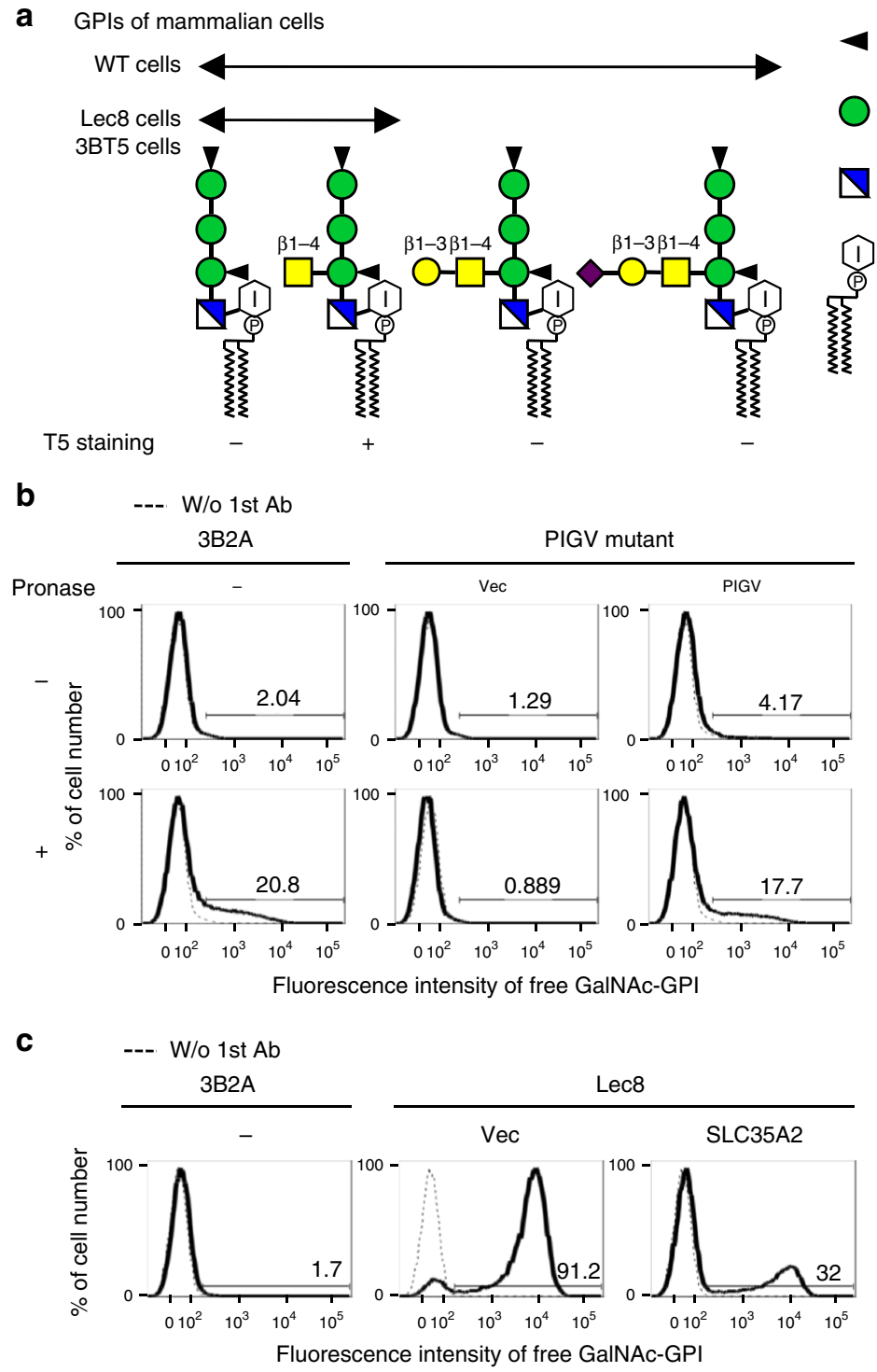

d -.- W/o 1 st $A b$
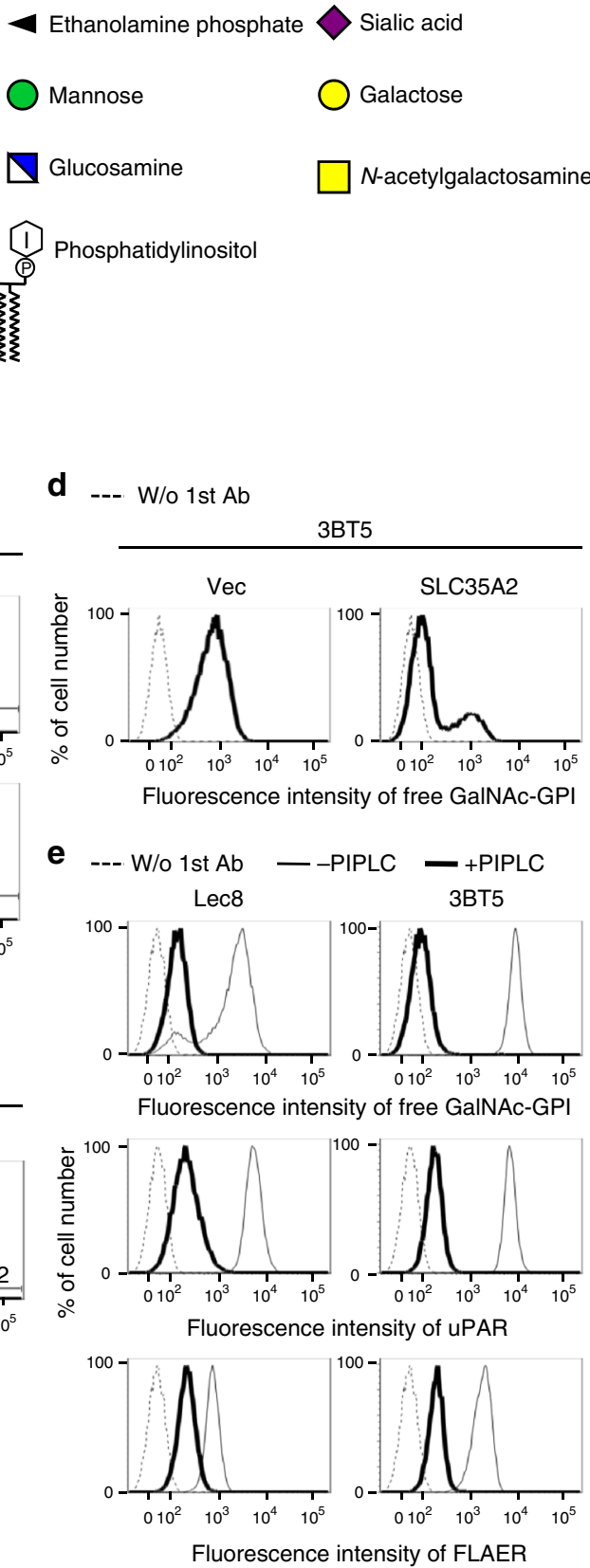

Fig. 1 Detection of GalNAc side-chain of mammalian free GPIs by T5 mAb. a GPI structures in wild-type (WT), Lec8 and 3BT5 CHO cells. - and +, T5 staining negative and positive, respectively. b 3B2A (WT) cells (left) and PIGV-mutant CHO cells transiently transfected with empty vector (center) or PIGV cDNA (right) were treated with (bottom) or without (top) pronase and then stained with T5 mAb and polyclonal second antibody. c Staining of Lec8 cells with T5 mAb. 3B2A (WT) cells and Lec8 cells transiently transfected with empty vector or SLC35A2 cDNA were stained with T5 mAb and second antibody. d Staining of 3BT5 cells transiently transfected with vector or SLC35A2 with T5 mAb and monoclonal second antibody. e GPI specificity of T5 $\mathrm{mAb}$. Cells were stained with T5 mAb, anti-uPAR, or FLAER with or without PI-PLC treatment. b-e Experiments were repeated at least twice. Dotted lines, background staining without first antibody. See also Supplementary Fig. 1 
specific membrane domains termed lipid-rafts or lipidmicrodomains. If GPI remodeling is disrupted, proteins containing abnormal GPI structures are expressed on the cell surface. Mutations in GPI biosynthetic and remodeling enzymes in humans cause several clinical symptoms, including intellectual disability, epilepsy, and abnormal facial features ${ }^{7}$. This demonstrates clearly that the correct GPI structure is functionally important. Thus understanding the remodeling pathway of GPI is important for revealing the biological significance of GPI anchoring.

In some GPI-APs, GPI can be further modified by glycan sidechains. In mammalian cells, the fourth Man can be attached to the third Man via an $\alpha 1-2$ linkage, by an ER-resident mannosyltransferase $\mathrm{PIGZ}^{8,9}$. $\mathrm{N}$-acetylgalactosamine (GalNAc) can be attached to the first Man via a $\beta 1-4$ linkage ${ }^{10}$. The GalNAc residue may be elongated by a $\beta 1-3$ linked galactose (Gal) and the Gal by sialic acid (Sia) (Fig. 1a) ${ }^{11}$. The addition of glycan sidechains gives structural diversity to GPIs. The physiological roles of these side-chains, however, are largely unknown because the enzymes for GalNAc-initiated side-chain addition have remained unknown since the existence of the side-chain was reported in $1988^{10}$. Recently, a pathological role of the GPI-GalNAc sidechain was suggested. Prions with a desialylated GPI inhibit the expansion of the disease form of prion $\left(\mathrm{PrP}^{\mathrm{sc}}\right)$ and also decrease the amount of $\mathrm{PrP}^{\mathrm{sc} 12}$. This indicates that sialylation of GPI contributes to prion diseases and that enzymes involved in the addition of the GPI-GalNAc side-chain represent drug targets. However, clarification of the biosynthetic pathway of the GPIGalNAc side-chain is required to understand the significance of the sialylation of GPI in vivo.

Glycosyltransferases (GTs) play pivotal roles in generating complex glycans ${ }^{13}$. Currently, GTs are classified into 103 families (in the Carbohydrate-Active Enzymes database) based on their amino acid sequence similarity. Interestingly, despite this diversity, the 3D structures of GTs fall into only three structural folds: GT-A, GT-B, and GT-C ${ }^{14}$. Almost all GT-A and GT-B enzymes that are localized in the Golgi exhibit the type II topology with an $\mathrm{N}$-terminal short cytoplasmic peptide, a single transmembrane domain (TMD), a stem region, and the GT fold ${ }^{15}$. These enzymes use nucleotide-sugars as donor substrates. One noteworthy characteristic of most GT-A enzymes is the DXD motif involved in the coordination of a divalent cation that is essential for binding the nucleotide-sugar ${ }^{14,16}$.

In this report, we aim to progress our understanding of the biosynthetic pathway of GPI-GalNAc side-chain. To this end, the identification of a $\beta 1-4-G a l N A c$ transferase, termed PGAP4, is presented. PGAP4 is a Golgi-resident GT with the GT-A fold and surprisingly has three TMDs. Results of homology modeling show that the GT-A fold of PGAP4 is split by tandem TMDs, positioning a catalytic cavity near the membrane to accommodate GPI glycolipid. Our findings uncover the initial step of GPIGalNAc biosynthesis and provide a platform to address the biological significance of this modification. Moreover, the identification of a GT with a unique topology expands the structural repertoire of the Golgi-resident GTs.

\section{Results}

T5 antibody recognizes mammalian free GPI modified by GalNAc. Previously, monoclonal antibodies (mAbs) were generated against free GPIs, i.e., non-protein linked GPIs, of the parasite Toxoplasma gondii ${ }^{17}$. T. gondii-free GPI has the $\beta \mathrm{Gal}$ NAc side-chain linked to the 4-position of the first Man and the GalNAc can be modified by glucose $(\mathrm{Glc})^{18}$. One of the antibodies, termed T5-4E10 mAb, hereafter "T5", was reported to detect the GalNAc side-chain lacking $\mathrm{Glc}^{18}$. We tested whether it is useful as a probe for the GPI-GalNAc side-chain in mammalian cells by assessing the immunoreactivity of $\mathrm{T} 5 \mathrm{mAb}$ in Chinese hamster ovary ( $\mathrm{CHO})$ cells by flow cytometry. We observed weak but measurable staining with $\mathrm{T} 5 \mathrm{mAb}$ after pronase treatment (Fig. 1b). The staining was not detected in a mutant $\mathrm{CHO}$ cell line defective in the PIGV gene, one of the GPI biosynthetic genes ${ }^{19}$, thereby confirming the specificity of T5 $\mathrm{mAb}$ for GPI (Fig. 1b). Importantly, we found that staining with $\mathrm{T} 5 \mathrm{mAb}$ was very high in Lec 8 cells ${ }^{20}$ even without pronase treatment. Lec8 is a mutant cell line defective in Gal addition to the GalNAc because of a defect in SLC35A2, which encodes the UDP-Gal transporter (Fig. 1a, c). Staining with T5 mAb was efficiently eliminated by phosphatidylinositol-phospholipase C (PI-PLC), which specifically cleaves GPI (Fig. 1e). These results showed that $\mathrm{T} 5 \mathrm{mAb}$ specifically recognizes terminally exposed GalNAc linked to free GPIs and that a significant amount of free GPIs bearing the GalNAc side-chain should be present on the $\mathrm{CHO}$ cell surface.

Forward genetic screening of GPI-GalNAc transferase. To identify the GPI-GalNAc transferase, we took a forward genetic approach in which randomly mutagenized SLC35A2-defective $\mathrm{CHO}$ cells were screened for T5 mAb staining-negative cells. The Lec8 cell was not suitable for this approach, because its culture always contains some cells that are not stained by T5 (Fig. 1c and Supplementary Fig. 1a). We first established the 3BT5 cell line containing only a negligible fraction of cells that were negative for T5 staining (Supplementary Fig. 1a). 3BT5 had non-functional SLC35A2, which was confirmed by the following experimental results: (1) 3BT5 cells were, like Lec8 cells, heavily stained with GSII or HPA lectins (Supplementary Fig. 1b); (2) staining with T5 $\mathrm{mAb}$ decreased when SLC35A2 was expressed (Fig. 1d) and (3) a F264S mutation was identified in SLC35A2 gene (Supplementary Fig. 1c). Finally, T5 staining of 3BT5 cells was abolished by PIPLC (Fig. 1e). Thus 3BT5 cells were used in the following experiments.

For random mutagenesis, 3BT5 cells were infected by a retrovirus harboring a gene-trapping plasmid ${ }^{21,22}$, followed by screening with $\mathrm{T} 5 \mathrm{mAb}$. We repeated cell sorting three times to enrich cells that were negative for T5 staining (see Methods section), resulting in the significant enrichment of mutant cells lacking T5 mAb staining (Supplementary Fig. 2a). To determine the insertion sites of gene-trapping vectors, genomic DNA was prepared from mutant cells before and after cell sorting, followed by deep sequencing. We ranked the mapping efficiency by reads per kilobase of exon per million mapped reads (RPKM) scores, which correspond to a density of sequence reads mapped to exons of a gene, and identified that TMEM246 (known as C9orf125 in humans), a previously uncharacterized gene, was greatly enriched in cells sorted three times (S3 cells) (Supplementary Fig. 2b). We termed TMEM246 as PGAP4 (post-GPI attachment to proteins 4). PGAP4 is widely conserved among species including Caenorhabditis elegans (F35C11.4, sequence ID: NP_495738) but surprisingly not in $T$. gondii. According to BioGPS, the mRNA expression of PGAP4 is limited to specific tissue, including the brain and spinal cord in both humans and mice, and the stomach, heart, and testes in mice ${ }^{23}$.

PGAP4 is essential for generating the GPI-GalNAc side-chain. To examine whether PGAP4 is required for GPI-GalNAc modification, a PGAP4 knockout $(\mathrm{KO})$ cell line of 3BT5 was generated by CRISPR-Cas924, 25. PGAP4-KO cells barely stained with T5 $\mathrm{mAb}$, indicating that GalNAc-modified free GPIs were lost (Fig. 2a). We then used matrix-assisted laser desorption ionization-time of flight (MALDI-TOF) mass spectrometry to 
determine the structure of protein-bound GPI. Because CD59 is known to have GalNAc on $\mathrm{GPI}^{26}$, we analyzed tagged-CD59 purified from PGAP4-KO cells transfected with PGAP4 cDNA or vector

and wild-type 3B2A cells. A fragment corresponding to GPI without $\mathrm{N}$-acetylhexosamine (HexNAc representing GalNAc) $(m / z=2531)$ was obtained from all three cell types, whereas the fragment corresponding to GPI with HexNAc $(m / z=2734)$ was detected only in PGAP4-transfected $\mathrm{KO}$ cells and wild-type cells (Fig. 2b). Liquid chromatography-electrospray ionization-tandem mass spectrometry (LC-ESI-MS/MS) analysis confirmed these structures (Supplementary Fig. 3) and showed that $>80 \%$ of GPI was modified by GalNAc in wild-type or PGAP4-rescued KO cells, whereas GalNAc modification was hardly detectable in PGAP4-KO cells (Fig. 2c and Supplementary Table 2). Therefore,

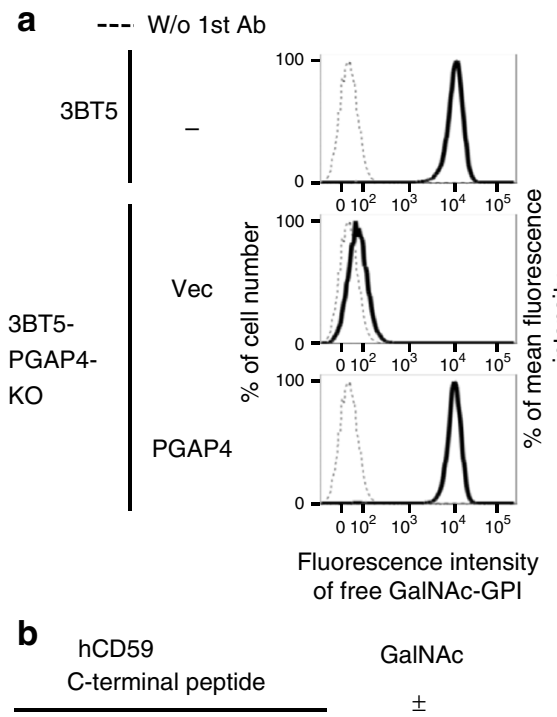

DLCNFNEQLEN-EtN-P-Man-Man-Man-GIcN-Ino-P

-GalNAc: $m / z=2530.9$

+GalNAc: $m / z=2733.9$

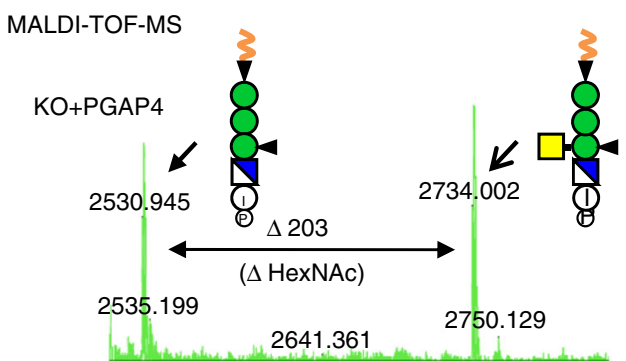

$\mathrm{KO}+\mathrm{Vec}$
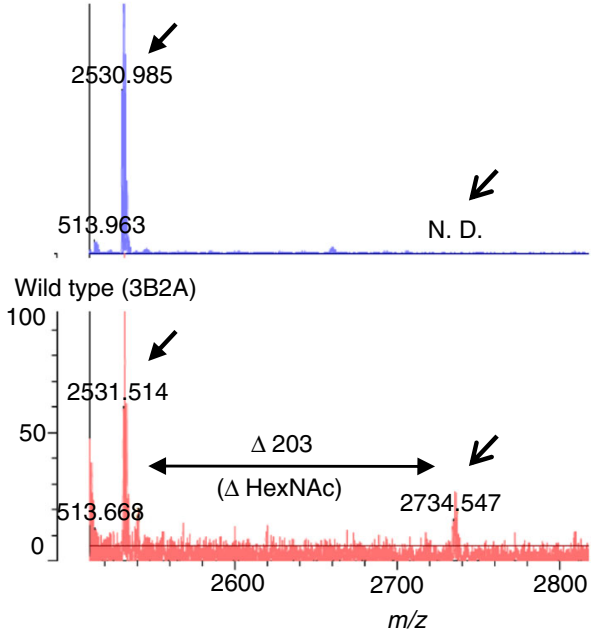

d
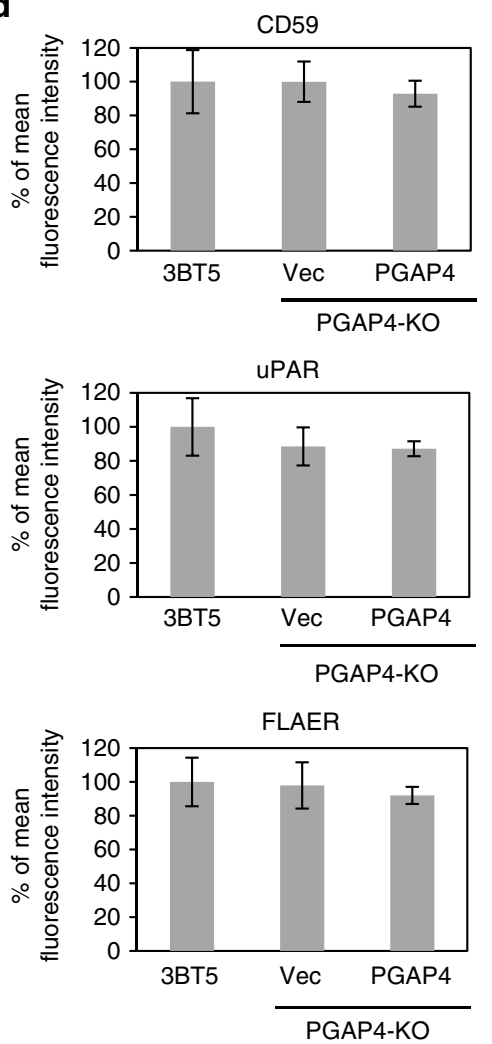

e

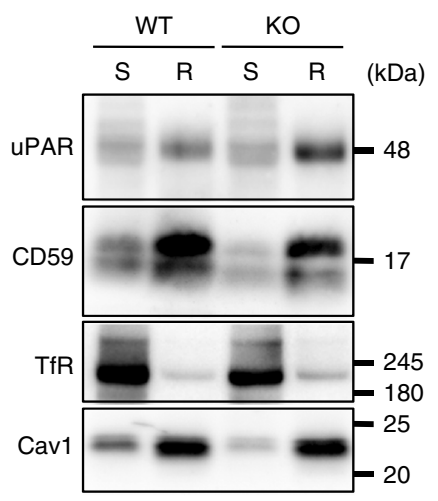

$\mathrm{S}$ : Triton soluble ; R : Triton resistant

C

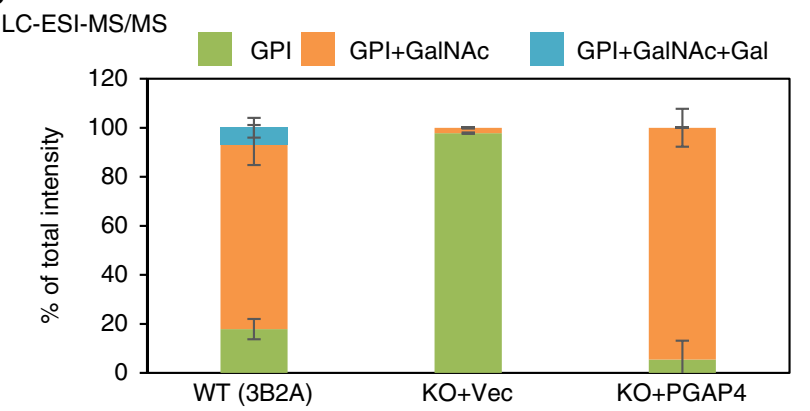


PGAP4 is essential for generating the GalNAc side-chain of both free and protein-bound GPIs.

In PGAP4-KO cells, surface expression of GPI-APs was not altered (Fig. 2d). In addition, the GalNAc side-chain was not involved in raft association of GPI-APs, as GPI-APs were still localized in the detergent-resistant membrane (DRM) in PGAP4-KO cells (Fig. 2e). These results suggest that the GalNAc side-chain does not affect the fundamental nature of GPI.

PGAP4 is a Golgi-resident GalNAc transferase with three TMDs. PGAP4 is predicted to be a transmembrane protein with one $\mathrm{N}$-glycosylation site and three TMDs (Fig. $3 \mathrm{a}$ and Supplementary Fig. 4a-c). Western blotting with Endo H or PNGase F treatment indicated that PGAP4 has a complex-type $N$-glycan (Fig. 3b). Immunofluorescent imaging revealed that PGAP4 was colocalized predominantly with GPP130, suggesting that PGAP4 is a Golgi membrane protein (Fig. 3c). DRM separation analysis revealed that PGAP4 is not incorporated into the raft fraction (Fig. 3d).

To examine PGAP4 topology experimentally, we artificially introduced an $\mathrm{N}$-glycosylation site into the PGAP4 amino acid sequence (Fig. 3e, upper). To eliminate the native $\mathrm{N}$-glycosylation site (N87), we first mutated this residue to A (N87A), and this mutant completely lost $\mathrm{N}$-glycosylation (Fig. 3e, lower). Two potential mutants with new $\mathrm{N}$-glycosylation sites were then generated, PGAP4 (N87A, F283N) and (N87A, T347N) (Fig. 3e, upper). T347N was $N$-glycosylated, whereas $\mathrm{F} 283 \mathrm{~N}$ was not (Fig. 3e, lower). Although it is known that some potential $\mathrm{N}$-glycosylation sites in the lumen are left unglycosylated, this result together with the computational topology prediction (Supplementary Fig. 4c) suggests that PGAP4 has three TMDs (TM1, TM2, and TM3), and that the region between TM1 and TM2 and the C-terminus face the lumen.

A homology search by PSI-BLAST using the PGAP4 amino acid sequence hit the $\mathrm{N}$-acetylglucosamine (GlcNAc) transferase, GnT-IV (Supplementary Fig. 5a). GnT-IV transfers GlcNAc to the Man of $N$-glycan via a $\beta 1-4$ linkage and PGAP4 is required for the addition of GalNAc to Man of GPI via a $\beta 1-4$ linkage (Supplementary Fig. 5b). Therefore, these two proteins may be expected to have similar structure. We found that GnT-IV and PGAP4s from various species have a conserved EDD motif similar to the DXD motif that is functionally important for many GTs in binding donor substrates (Supplementary Fig. 5a, c). These results support the idea that PGAP4 is a Golgi-resident GalNAc transferase.

PGAP4 has a GT-A fold split by an insertion of tandem TMDs. To understand the structure-function relationship of PGAP4, a three-dimensional (3D) structural model of PGAP4 was predicted. Due to a lack of the significant homology with any known 3D structure available in the Protein Data Bank, comparative modeling of full-length PGAP4 could not be achieved. Instead, multiple threading approach using the PGAP4 amino acid sequence lacking TM2 and TM3 (PGAP4DTM2, TM3) identified sequence similarity with bacterial cellulose synthase (Protein Data Bank (PDB) ID: 4P02 27 ) (Supplementary Fig. 6). Hence, we first used a fold recognition approach, Iterative Threading ASSEmbly Refinement (I-TASSER) ${ }^{28}$, to construct an atomic model of PGAP4 $\triangle T M 2$, TM3 using the crystal structure of bacterial cellulose synthase (PDB ID: 4P02A) as the template structure. The overall quality of the predicted model was analyzed by PROSA$\mathrm{Web}^{29}$ and RAMPAGE ${ }^{30}$ as described in the Methods section, which indicated that the model structure is of comparable quality to low-resolution experimental structure of the similar size. The model shows that PGAP4ATM2, TM3 consists of a short tail (residues 1-19), the N-terminal TMD (TM1), a luminal helical region (residues 43-82), and a luminal core domain (residues 83-261 and 309-403). The modeled PGAP4DTM2, TM3 aligns with the catalytic domain and the preceding helical region of the cellulose synthase (Fig. 4a). The luminal core domain of PGAP4DTM2, TM3 consists of a seven-stranded $\beta$-sheet surrounded by $\alpha$-helices, aligning well with the GT-A fold of the cellulose synthase (Fig. 4a). Within the GT-A domain of PGAP4DTM2, TM3, the N-terminal part and the C-terminal part closely interact with each other (Fig. $4 \mathrm{~b}$ ). The fold similarities detected by I-TASSER suggest that PGAP4 contains the GT-A fold separated by tandem TMDs. The luminal helical region between TM1 and the GT-A domain corresponds to a stem region in typical Golgi GTs of type II topology (Fig. 4b).

Validated PGAP4 3D structure with three TMDs. The PGAP4 sequence contains eight conserved cysteine residues in the luminal regions (Supplementary Fig. 5c), indicating the possibility of disulfide bond formation. An atomic model of the luminal core domain of PGAP4 $(\Delta 1-19, \Delta 262-308)$ stipulates for three pairs of cysteine residues, C132-C136, C144-C194, and C332-C333, that are sufficiently close to each other to form disulfide bonds (C $\alpha$ distances: 10.2, 8.9, and $3.8 \AA$ for C132-C136, C144-C194, and C332-C333, respectively) (Supplementary Fig. 7a). To validate these disulfide pairs, mass spectrometry of purified PGAP4 was conducted. The result confirmed the presence of three disulfide bridges, C132-C136, C144-C194, and C332-C333 (Supplementary Fig. 7b, c). The two remaining cysteines, C43 and C356, are located quite far from each other and cannot interact in the model. Mutational analysis confirmed that C43 and C356 are in free (reduced) form (Supplementary Fig. $7 \mathrm{~d}-\mathrm{g}$ ). These lines of experimental evidence are consistent with the model of PGAP4, supporting that the 3D structural model of PGAP4 is valid. Finally, we used the structure of the PGAP4 GT-A fold and the 3D structure of cellulose synthase as templates to model the overall PGAP4 structure with three TMDs using a comparative modeling approach ${ }^{31}$. A series of distance

Fig. 2 Analysis of PGAP4-KO cells. a Left. 3BT5 CHO cells (top) and 3BT5-PGAP4-KO cells stably harboring pLIB2-Hyg (Vec) or pLIB2-Hyg-hPGAP4-3HA (PGAP4) were stained with T5 mAb. Dotted lines, background staining. Right. Mean fluorescence intensity ( \pm SD) from three independent experiments $(n=3)$. Statistical analyses were done by unpaired Student's $t$-test. ${ }^{\star} p<0.05 ;{ }^{\star \star} p<0.005 . \mathbf{b}$ and $\mathbf{c}$ A lack of GalNAc side-chain in GPI of CD59 in PGAP4$\mathrm{KO}$ cells. GPI-containing peptide from PI-PLC-treated CD59 was analyzed by MALDI-TOF-MS (b) or LC-ESI-MS/MS (c). b The C-terminal peptide containing GPI after trypsin digestion is shown (top). The $\mathrm{m} / \mathrm{z}$ of GPI-containing peptides with or without HexNAc are 2733.9 and 2530.9 , respectively. c Quantification of MS data. Percentage of total intensity (mean \pm SD) was calculated from the peak areas obtained by two independent measurements. $\mathbf{d}$ Normal levels of GPI-APs on PGAP4-KO cells. 3BT5 cells and 3BT5-PGAP4-KO cells stably harboring pLIB2-Hyg (Vec) or pLIB2-Hyg-hPGAP4-3HA (PGAP4) were stained with anti-CD59 (top), anti-uPAR (center) and FLAER (bottom). Mean fluorescence intensity ( \pm SD) from three independent experiments $(n=3)$. Statistical analyses were done by unpaired Student's $t$-test. Not significant. e DRM separation experiment. Transferrin receptor (TfR) and Caveolin1 (Cav1) are markers of Triton-soluble (S) and -resistant (R) fractions, respectively. Representative data from two independent experiments. See also Supplementary Fig. 2, 3 


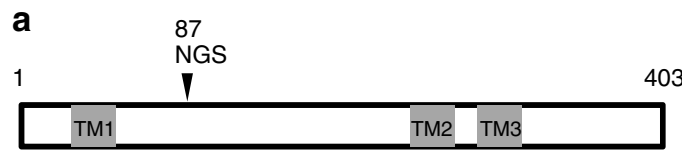

Transmembrane domain

C

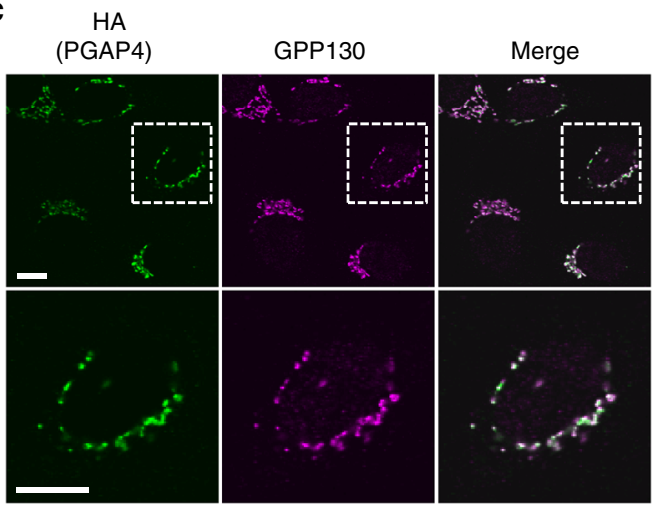

d

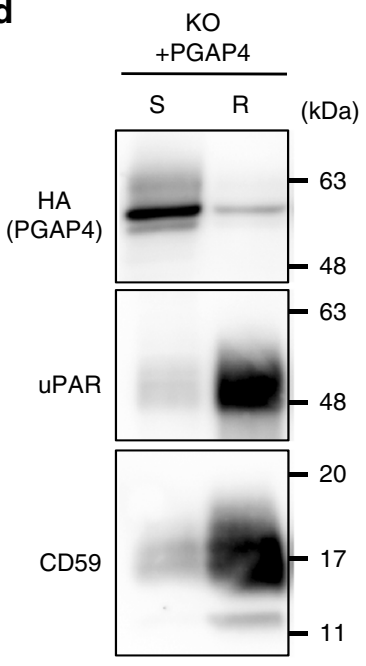

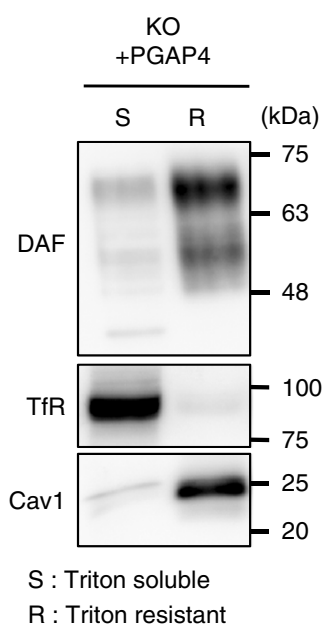

b

$$
\begin{array}{lllll}
\text { PGAP4 } & - & + & + & + \\
\text { Endo-H } & - & - & + & -
\end{array}
$$$$
\text { PNG-F } \quad-\quad-\quad-\quad+\quad(k D a)
$$

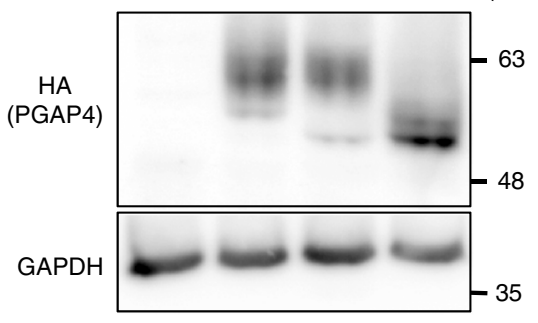

e
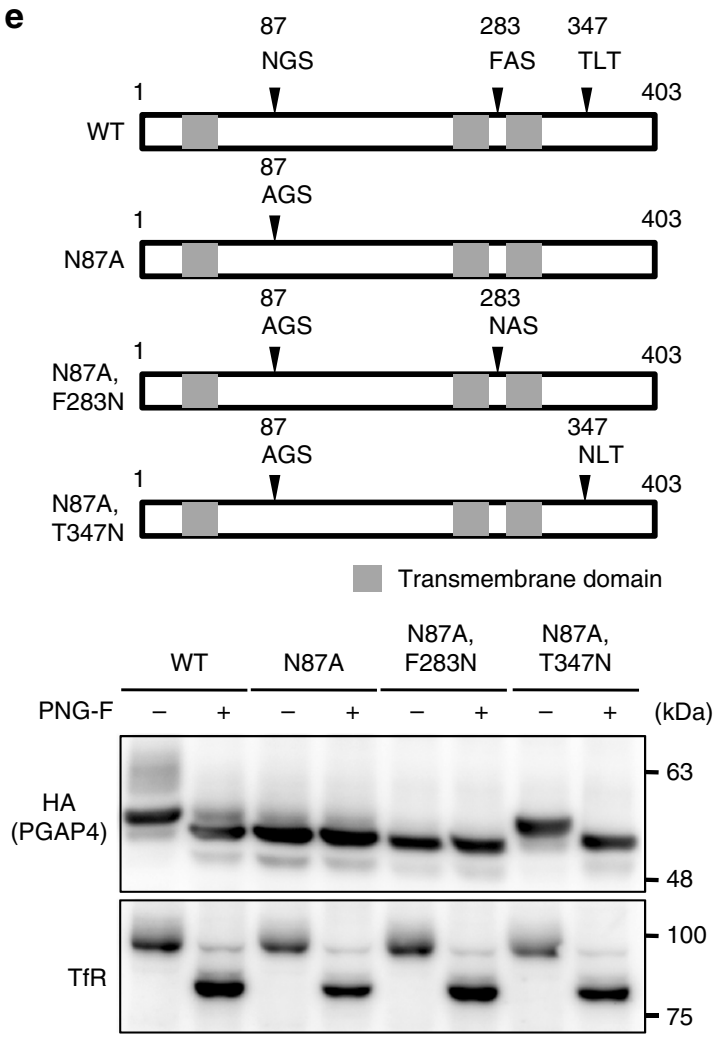

Fig. 3 Characterization of PGAP4. a Schematic of PGAP4 protein. Arrowhead, an N-glycosylation site; gray boxes, transmembrane domains. b Western blotting of PGAP4. Lysates of 3B2A cells stably expressing PGAP4-3HA were treated with Endo H or PNGase F followed by western blotting. GAPDH, a loading control. c Immunofluorescence imaging of PGAP4. 3B2A was transiently transfected with pME-hPGAP4-3HA. Lower panels are enlarged images of regions surrounded by white squares in the upper panels. GPP130, the Golgi marker. Scale bars: $10 \mu \mathrm{m}$. d Non-DRM localization of PGAP4. Lysate of 3BT5PGAP4-KO cells transiently expressing PGAP4-3HA were fractionated into Triton-soluble (S) and -resistant (R) fractions and subjected to western blotting. e Analysis of PGAP4 topology. (Upper) Schematic of PGAP4 WT and N-glycosylation mutants. Arrowheads, target sites of mutations. (Lower) Western blotting of PGAP4 WT and mutants. pME-hPGAP4-3HA was transiently transfected in 3B2A cells. Lysates were treated with or without PNGase F. TfR, a positive control for PNGase F treatment. b-e Representative data from two independent experiments. See also Supplementary Fig. 4 and 5

restraints was used to assemble three TMDs in a plane and to incorporate the disulfide bonds in the model (see Methods section), generating a complete 3D structural model of PGAP4 (Fig. 4c, Supplementary Fig. 8, Supplementary Data 1, and Supplementary Movie 1).

Characterization of the catalytic region of PGAP4. Structural superposition of the GT-A fold in PGAP4 and the cellulose synthase indicates a putative cavity accommodating a donor substrate UDP-GalNAc (Fig. 4d). The DXD motif (D246-A247-D248) of the cellulose synthase, which coordinates an $\mathrm{Mg}^{2+}$ ion for UDP-Glc binding, is superimposable with the E211-D212-D213 of PGAP4. To determine the functional significance of this motif, PGAP4 mutants E211A and D213A were constructed. As expected, both mutants completely lost the activity to restore GalNAc side-chain in 3BT5-PGAP4-KO cells (Fig. 4e-g). These results demonstrate that E211-D212-D213, a DXD-like motif, is essential for the activity of PGAP4.

The catalytic center of the cellulose synthase, D343, which is close to the DXD motif, corresponds to the D363 in PGAP4, which is conserved among species (Fig. 4d and Supplementary Fig. 5c). A D363A mutant of PGAP4 also lost the activity (Fig. $4 \mathrm{~h}-\mathrm{j}$ ), indicating D363 to be a putative catalytic center of this enzyme.

Mutational analyses highlight the UDP-GalNAc-binding site. To simulate the binding mode of PGAP4 to a donor substrate, we incorporated UDP-GalNAc into the model by adopting UDP-Glc from the cellulose synthase structure during the homology 
modeling (Fig. 5a). Residue D363 is located near the GalNAc where it can mediate catalysis (Fig. 5a, b and Supplementary Movie 1). The model shows that V109, T334, P335, and K362, all of which are conserved residues, form a cavity for UDP-GalNAc (Fig. 5b and Supplementary Fig. 5c). Activities of V109A, P335A, and $\mathrm{K} 362 \mathrm{~A}$ mutants were reduced significantly, and the T334A mutant was almost inactive; all these mutants showed the proper Golgi localization and wild-type-level expression (Fig. 5c-e). These results indicate that all four residues form a cavity that accommodates UDP-GalNAc.
PGAP4 may interact with GPI at a juxtamembrane region. The PGAP4 model demonstrated that the enzyme has a concave surface in the juxtamembrane region near the catalytic site (Fig. 6a and Supplementary Movie 1). The open space between this surface and the membrane seems sufficiently wide to accommodate the GPI glycan (Fig. 5a). To investigate the functional significance of the concave surface, mutational analysis was performed on six residues, H247, E249, M260, H311, F313, and R317, located on this surface (Fig. 6a and Supplementary Fig. 5c and Supplementary Movie 1). The activities of the H247A and
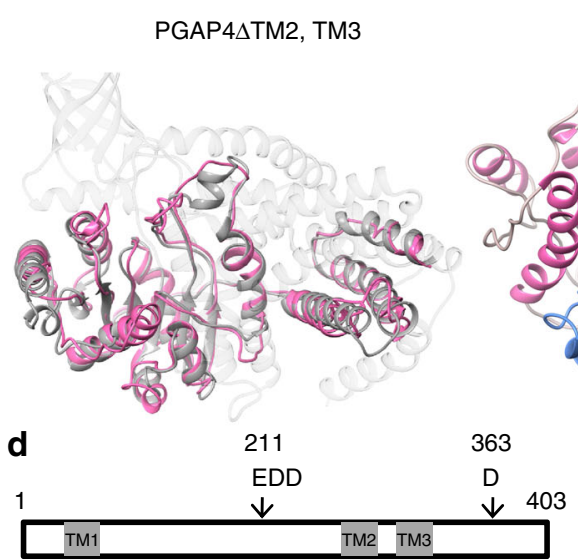

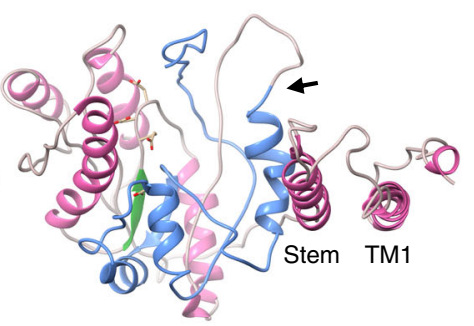

b

403 c $\mathrm{C}_{144-\mathrm{C} 194} \mathrm{C} 132-\mathrm{C} 136$

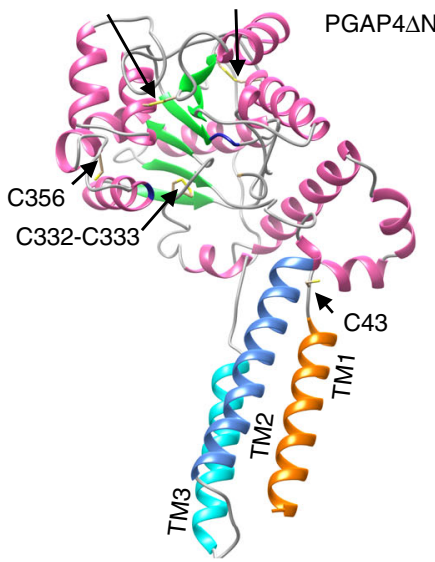

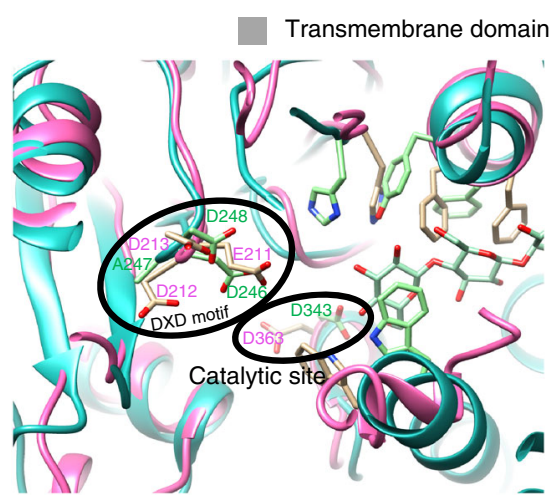

g

g --- W/o 1st Ab
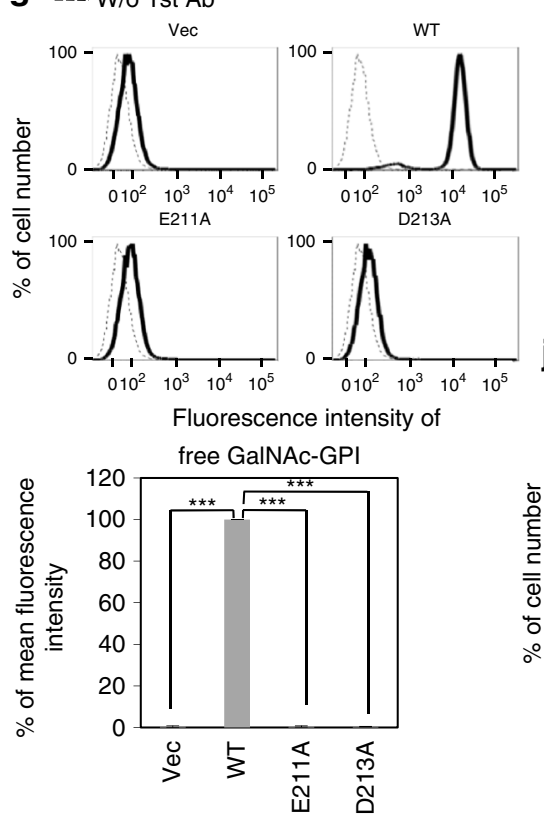

e

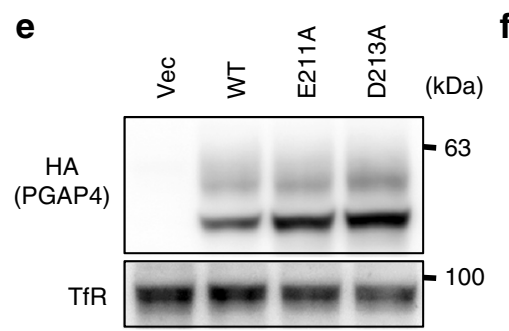

f
ET
E211A

HA

(PGAP4) GPP130 Merge

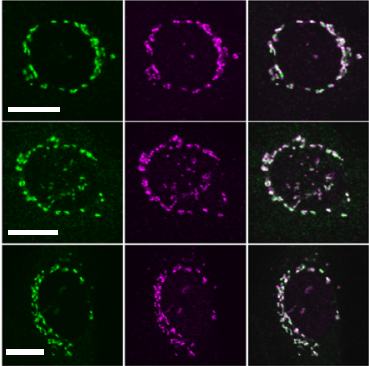

h

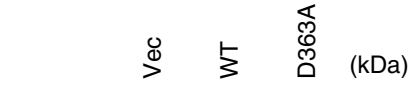

$\mathbf{i}$

\section{i}
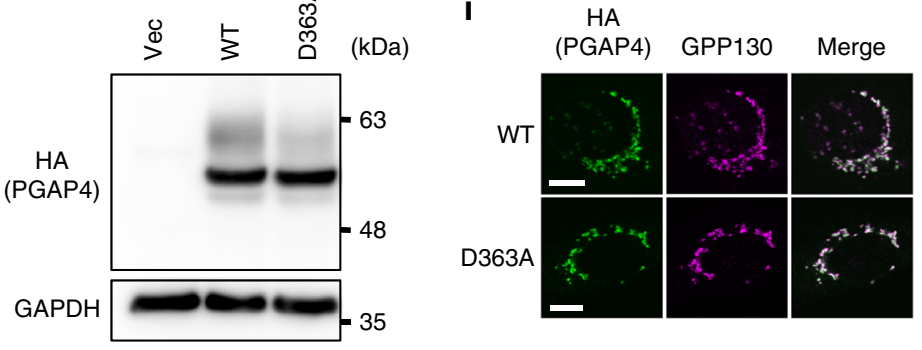

j

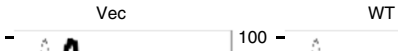

-
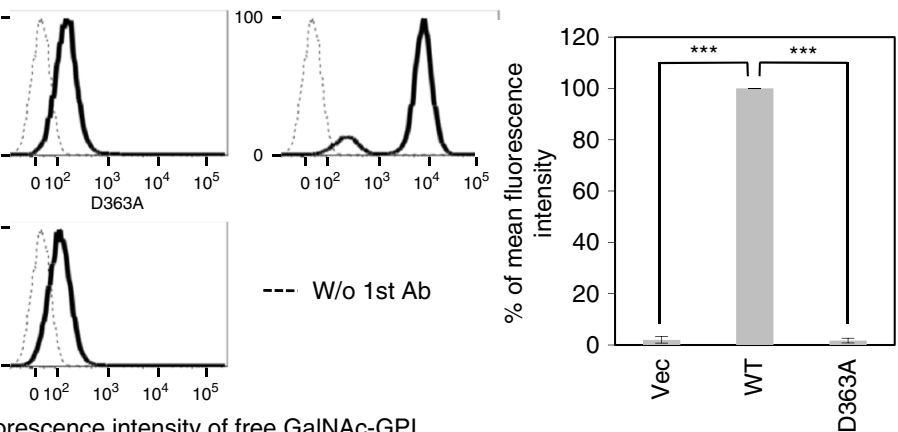
F313A PGAP4 mutants were significantly reduced without affecting the expression or localization (Fig. 6b-d). E249A mutation, but not H311A and M260A, weakly but significantly reduced the activity (Fig. 5c-e and Supplementary Fig. 9). R317A mutation severely affected the activity due to accumulation of this mutant in the ER (Supplementary Fig. 9). Combined with the knowledge gained from the structural model, these results suggest that the concave surface in the juxtamembrane region of PGAP4 is involved in the recognition of the GPI glycan (Fig. 6e). Mutations in TM2 (M270A) and TM3 (M302A) did not alter the enzyme activity (Fig. 5c, e and Supplementary Fig. 9). However, because the TMDs are adjacent to the concave surface, we propose that PGAP4 interacts with the GPI lipid via one or more of the three TMDs (Fig. 6e).

The N-terminal TMD of PGAP4 is the Golgi targeting signal. The N-terminal TMD of many Golgi-resident GTs acts as a Golgi-targeting signal ${ }^{32}$. To determine whether TM1 of PGAP4 acts as a Golgi-targeting signal, both the N-terminal cytoplasmic domain and TM1 were deleted (PGAP4DTM1). We found that PGAP4 $\Delta$ TM1 accumulated in the ER (Fig. 6f). We then made a green fluorescent protein (GFP) fusion with TM1 of PGAP4 (TM1-GFP). TM1-GFP colocalized with GPP130 (Fig. 6g). These results indicate that TM1 of PGAP4 functions as a signal for Golgi targeting, as seen for other GTs.

Substrate specificity and timing of GalNAc transfer. To investigate the acceptor substrate specificity of PGAP4, we used mutant $\mathrm{CHO}$ cell lines each defective in one of the GPIremodeling enzymes. The cells were also made defective in SLC35A2 to prevent Gal addition to GalNAc, which interferes with probing with T5 mAb. We analyzed the GPI anchor of CD59 by mass spectrometry and/or free GPIs by T5 staining.

EtNP on the first Man is attached by the enzyme PIGN and therefore loss of PIGN activity leads to the loss of EtNP on the first Man (Fig. 7a) ${ }^{33}$. The PIGN-SLC35A2-defective cells partially lost the surface expression of CD59 as expected (Fig. 7b) ${ }^{33}$. However, staining with $\mathrm{T} 5 \mathrm{mAb}$ was comparable to that of wildtype cells (Fig. 7b). Overexpression of PIGN in the PIGNSLC35A2-defective cells normalized the CD59 expression, but it did not enhance the T5 mAb staining (Supplementary Fig. 10a). These results indicate that the EtNP side-chain on the first Man is dispensable for recognition by PGAP4 and that it is not part of the epitope recognized by the $\mathrm{T} 5 \mathrm{mAb}$.
PGAP5 removes EtNP from the second Man before GPI-APs exit the ER. Therefore, PGAP5-defective cells have three EtNPs in GPI-APs, whereas wild-type cells have two EtNPs (Fig. 7a) ${ }^{3}$. PGAP5-SLC35A2-defective cells (C19-SLC35A2-KO) were generated accurately as indicated by a similar lectin staining pattern to that of Lec8 cell lines (Supplementary Fig. 10b). Mass spectrometry revealed that the amount of GalNAc modification of CD59 in PGAP5-SLC35A2-defective cells was not reduced (Fig. 7c, Supplementary Fig. 11, and Supplementary Table 3). These results indicate that removal of the EtNP from the second Man is not required for substrate recognition by PGAP4.

GPI-APs undergo fatty acid remodeling in the Golgi (Fig. 7e $)^{5,6}$. We asked whether GalNAc transfer by PGAP4 occurs before or after fatty acid remodeling. To answer this question, we generated a PGAP2-KO line from 3BT5 cells. PGAP2-defective cells produce GPI-APs bearing the lyso-form of GPI and transport these GPI-APs on the cell surface (Fig. 7a). Proteins bearing the lyso-form GPI have only one hydrocarbon chain for their membrane insertion and are released from the membrane spontaneously or after cleavage by GPI phospholipase $\mathrm{D}^{6}$. If GalNAc transfer occurs before the fatty acid remodeling, the fraction of GalNAc-modified GPI may be similar in GPI-APs in wild-type cells and those released into the medium from PGAP2-KO cells. If GalNAc transfer occurs after fatty acid remodeling, a fraction of GalNAc-modified GPI might be smaller in proteins released from PGAP2-KO cells because of altered GPI lipid structure. Experimentally, PGAP2-KO cells showed dramatically reduced surface expression of GPI-APs and T5 mAb staining, which were restored by transfection of PGAP2 cDNA, confirming the successful KO (Supplementary Fig. 10c). Mass spectrometry revealed that the efficiency of GalNAc modification was greatly decreased in PGAP2-KO cells (GPI + GalNAc; $95.5 \% \pm 0.68 \%$ [WT] vs. $74.1 \% \pm 0.13 \%$ [PGAP2-KO]) (Fig. 7d, Supplementary Fig. 12, and Supplementary Table 4). Thus we concluded that PGAP4 functions after fatty acid remodeling (Fig. 7e).

Finally, we used PGAP3-KO cells to test whether the fine structure of the $s n$-2-linked fatty acyl chain in GPI is critical for recognition by PGAP4. Whereas wild-type cells mostly have a saturated fatty acid (stearic acid) at the $s n-2$ position, PGAP3-KO cells have an unsaturated fatty acid (such as arachidonic acid or docosatetraenoic acid) at the $s n-2$ position, and because of this abnormality the GPI-APs do not efficiently associate with the $\mathrm{DRM}^{5,6}$. We generated a PGAP3-KO line from 3BT5 cells. The DRM separation experiment confirmed the successful $\mathrm{KO}$ of

Fig. 4 3D structural modeling of PGAP4 demonstrating the unique split GT-A fold. a 3D structural model of PGAP4 lacking two TMDs (TM2 and TM3, corresponding to 262-308). The structure of PGAP4DTM2, TM3 (pink) is superimposed to GT domain of the crystal structure of cellulose synthase (dark gray, PDB: 4P02A). Non-GT regions of cellulose synthase are in light gray. b The structural model of PGAP4 $4 T M 2$, TM3 exhibited split GT-A fold. Pink region, a region $\mathrm{N}$-terminal to TM2; blue region, a region C-terminal to TM3; arrow, the joint after removal of two TMDs. c 3D structural model of PGAP4 with disulfide bridges and three TMDs displayed in ribbon model. PGAP4 $\Delta N(\Delta 1-19)$ is shown. Cysteine residues are displayed with sticks. $\mathbf{d}$ Enlarged view of superimposed catalytic regions. (Upper) Schematic of PGAP4. Arrows, DXD-like motif and putative catalytic site. (Lower) Functionally important residues are shown with sticks. Pink: PGAP4DTM2, TM3; cyan: cellulose synthase (PDB: 4P02A). Cellulose is displayed. e-g Analysis of DXD-like motif mutants of PGAP4 by western blotting (e), immunofluorescence imaging (f), and flow cytometry (g). pME-hPGAP4-3HA-bearing WT or mutant sequences was transiently transfected into 3BT5-PGAP4-KO cells and analyzed. e TfR, loading control. $\mathbf{g}$ Activities of PGAP4 mutants to restore GalNAcmodified GPI as assessed by staining with T5 mAb. (Upper) Dotted lines, background staining. (Lower) Quantitative data. Statistical analyses were done by unpaired Student's $t$-test. ${ }^{\star \star \star} p<0.0005$. h-j Analysis of catalytic mutant of PGAP4 by western blotting (h), immunofluorescence imaging (i), and flow cytometry (j). pME-hPGAP4-3HA-bearing WT or D363A sequence was transiently transfected into 3BT5-PGAP4-KO cells. h GAPDH, loading control. $\mathbf{i}$ GPP130, the Golgi marker. Scale bars: $10 \mu \mathrm{m}$. j Functional analysis of the catalytic site mutant. Cells were stained with T5 mAb. (Left) Dotted lines, background staining. (Right) Quantitative data. Statistical analyses were done by unpaired Student's $t$-test. ${ }^{\star \star *} p<0.0005$. e, $\mathbf{f}, \mathbf{h}, \mathbf{i}$ Representative data from two independent experiments. $\mathbf{g}$, $\mathbf{j}$ Mean fluorescence intensity $( \pm$ SD) from three independent experiments $(n=3)$. T5 staining of cells restored by WT PGAP4 was set as 100\%. See also Supplementary Fig. 5, 6, 7 and 8 
PGAP3 (Supplementary Fig. 10d). Flow cytometry revealed that T5 $\mathrm{mAb}$ staining decreased by $50 \%$ in the PGAP3-KO cells with no change in the expression of GPI-APs (Fig. 7f). However, mass spectrometry of protein-bound GPI showed a smaller difference in the efficiency of GalNAc modification (93.5\% [WT] vs. $86.2 \%$ [PGAP3-KO]) (Fig. 7g and Supplementary Table 5). These results suggest that the fine structure of the $s n$-2-linked acyl chain is not strictly recognized by PGAP4.

\section{Discussion}

We have characterized the first step in the biosynthetic pathway of the GalNAc side-chain of mammalian GPI. We used T5 mAb, which was developed against a side-chain of free GPIs from $T$. gondii ${ }^{17,18}$, as a probe for the GPI-GalNAc side-chain. T5 mAb requires terminally exposed GalNAc for its recognition of free GPIs. Although wild-type $\mathrm{CHO}$ cells are $\mathrm{T} 5$ staining negative, pronase treatment makes them T5 staining positive. Pronase
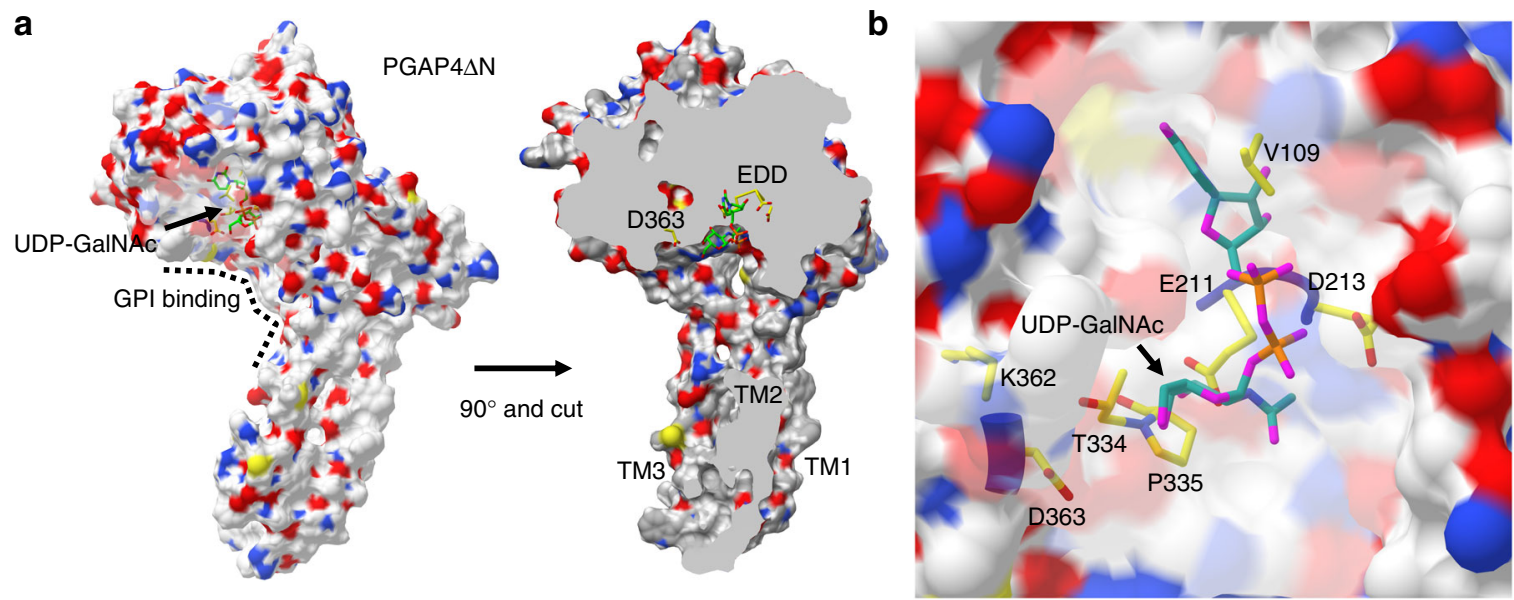

c

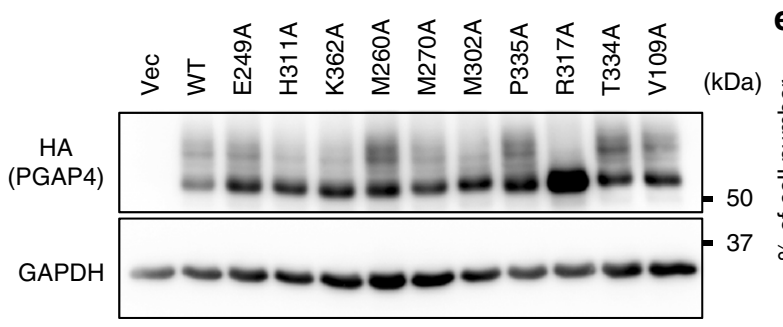

e

--- W/o 1 st $A b$ Vec WT

T334A
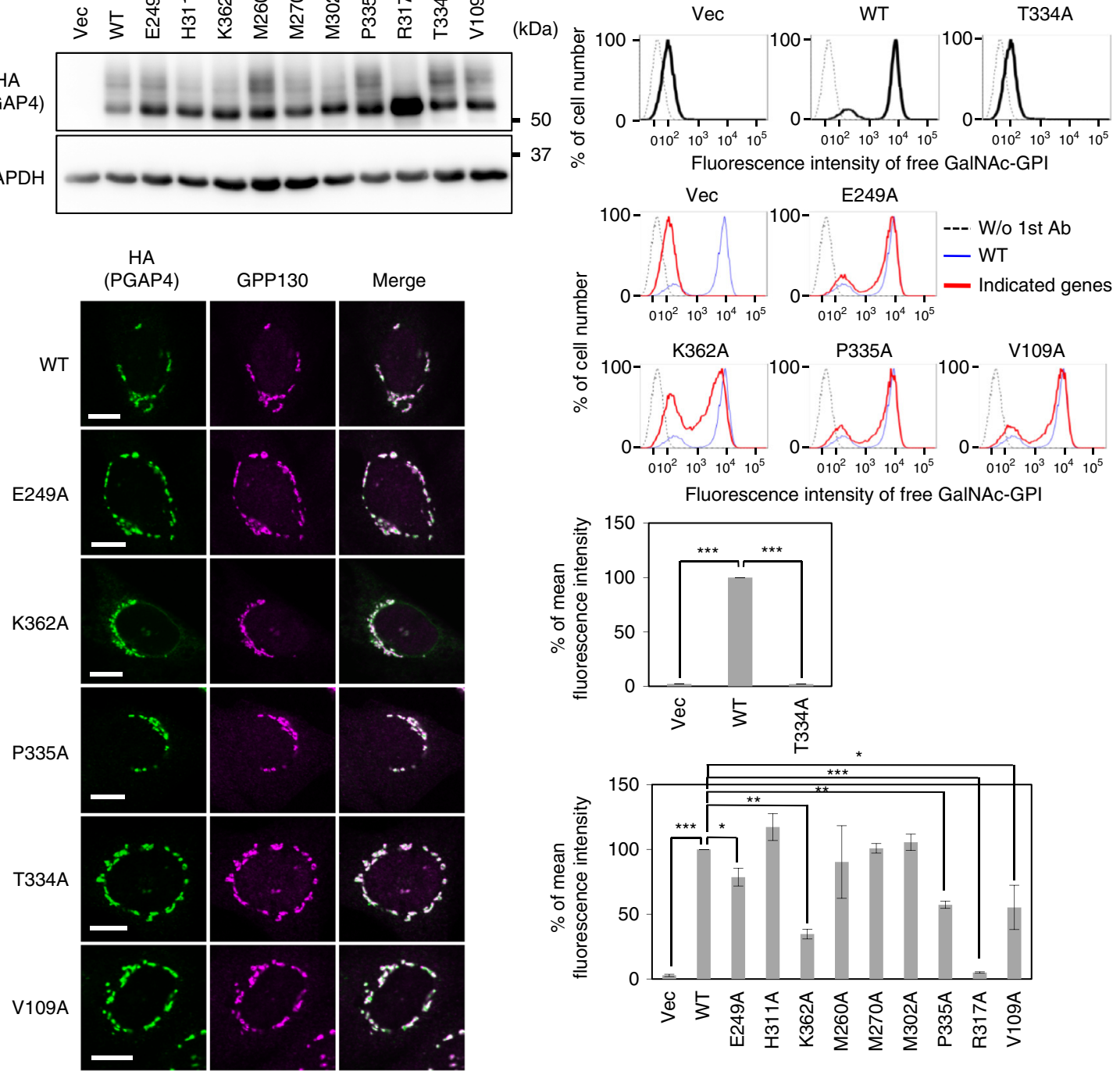

Fluorescence intensity of free GalNAc-GPI
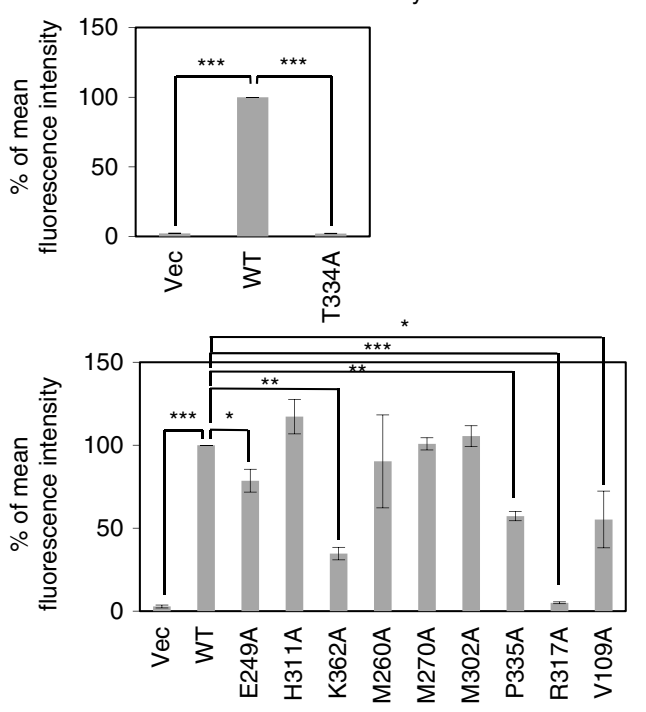
probably cleaves amide bonds between C-terminal residues and EtNP of GPIs ${ }^{34}$, thereby free GPIs are newly generated, suggesting that some GPI-APs have terminally exposed GalNAc sidechain. On the other hand, $\mathrm{CHO}$ Lec8 cells that are defective in UDP-Gal transport into the Golgi are clearly positive for T5 staining. CHO cells, therefore, have some free GPI, almost all of which is further modified with Gal.

We identified PGAP4 (originally termed TMEM246) as a GPImodifying GalNAc transferase in the Golgi. Our data demonstrate that GalNAc addition to GPI is carried out in the Golgi after fatty acid remodeling (Fig. 7e). This is consistent with the fact that GalNAc modification of various glycans occurs in the cis to medial Golgi ${ }^{35}$, whereas fatty acid remodeling may take place in the cis Golgi ${ }^{5,6}$. A lack of PGAP4 in CHO cells did not alter the fundamental nature of the GPI-APs, including surface expression levels and raft association (Fig. 2d, e). So, the biological role(s) of the GalNAc side-chain remain to be clarified.

Since typical Golgi-resident GTs are type II membrane proteins that have a short N-terminal peptide, one TMD, a luminal stem region, and a GT fold ${ }^{15}$, PGAP4 shows a unique modular architecture. 3D homology modeling of PGAP4 demonstrated that the structural arrangement of PGAP4 has a short N-terminal peptide, one TMD, a luminal stem region, and the GT-A fold with inserted tandem TMDs. This indicates that PGAP4 shares common structural properties with previously characterized Golgi-resident GTs, aside from the two TMD insertions. Consistent with this, the first TMD of PGAP4 functions as the Golgitargeting signal similar to other Golgi-resident GTs ${ }^{32}$ (Fig. 6f, g). Thus PGAP4 shows both unique and common features found in GTs. PGAP4 is a Golgi-resident GT that shows a split GT-A fold with multiple TMDs. Whether such structural topology exists for other GTs remains to be seen.

We have identified residues critical for enzymatic activity of PGAP4, including a DXD-like motif, the catalytic site, and the region that potentially recognizes GPI glycan. These residues are closely localized to each other, suggesting that reaction can be carried out at their interface. The three TMDs and the functionally essential residues are also close. Mass spectrometry in PGAP3-KO and PGAP2-KO cells indicates that PGAP4 does not distinguish the precise lipid structure of GPI but instead needs two fatty acid chains. Given the 3D structural model, we propose two significances of tandem TMD insertion: (1) to approximate the catalytic site to GPI at the vicinity of the membrane and (2) to interact with GPI lipid by at least one TMD. The unique topology of PGAP4 is suitable to accommodate the glycolipid substrate, GPI (Fig. 6e).

Using PSI-BLAST, PGAP4 orthologs were identified in various species but surprisingly not in T. gondii, even though that parasite has GPI with a GalNAc side-chain. A previous study showed that GalNAc modification was carried out in the ER in T. gondii ${ }^{36}$. These observations suggest that $T$. gondii uses a GalNAc transferase that is not orthologous to PGAP4. Identification of another GalNAc transferase is thus an aim of future study.
Previous studies revealed that not all GPI-APs are modified with a GalNAc side-chain. This indicates two possibilities. One is limited distribution of PGAP4 expression. The GalNAc side-chain was detected in some GPI-APs purified from brain, kidney and skeletal muscle $10,11,37,38$ but not from erythrocytes or placenta $^{39,40}$, consistent with the expression profile of PGAP4 reported in BioGPS. The other possibility is a structural constraint in PGAP4. Although GPI should be sufficient for the recognition by PGAP4 because free GPIs can be modified by GalNAc, some GPI-APs might escape from PGAP4. Based on our structural model, PGAP4 and the C-terminus of the target proteins could be in close proximity. Thus, 3D structure of the target proteins or some modifications to the C-terminal region, such as heavy glycosylation, may interfere with PGAP4 interaction. Further studies are needed to clarify this possible functional feature.

In conclusion, we have uncovered the initial step of the GalNAc modification of GPI. Our findings establish a genetic basis for studying the significance of the GPI-GalNAc side-chain. With this in mind, we are currently preparing PGAP4-KO mice, which will facilitate examination of the physiological and pathological significance of the GPI-GalNAc side-chain in vivo. Moreover, we have provided evidence that PGAP4 is a Golgi-resident GT with an unusual modular arrangement. This may provide a basis for identifying other enzymes with similar architectures, which were not considered to be GTs or remained uncharacterized so far.

\section{Methods}

Reagents and antibodies. Following reagents were purchased: Pronase (Roche); FLAER-AlexaFluor488 (Cedarlane); GSII-AlexaFluor647 and HPA-AlexaFluor647 (L32451 and L32454, Thermo Fisher); MAM-fluorescein isothiocyanate (FITC) (J510, J-OIL-MILLS); PI-PLC (P6466, Thermo Fisher); Imperial Protein Stain (24615, Thermo Fisher); RNeasy Mini Kit (74104, QIAGEN); SuperScript VILO Master Mix (11755050, Thermo Fisher); Complete, EDTA-free Protease Inhibitor Cocktail (11873580001, Roche); FLAG-M2 beads and 3× FLAG peptide (A2220 and F4799, Sigma-Aldrich); Glutathione Sepharose 4B (17075601, GE Healthcare); and glutathione (Reduced form, 073-02013, Wako). T5-4E10 (T5) was kindly gifted from Dr. J. F. Dubremetz (Montpellier University ${ }^{17}$ ). Mouse monoclonal anti-human CD59 (clone 5H8 ${ }^{41}$ ), anti-human DAF (clone IA10, BD, cat\# 565939), and anti-hamster uPAR (clone 5D6 ${ }^{41}$ ) were used. Antibodies for human CD59 and hamster uPAR are available from the authors upon request. Following antibodies were purchased: Mouse monoclonal anti-HA (clone HA7, Sigma-Aldrich, cat\# H3663), anti-Caveolin 1 (clone 2297, BD, cat\# 610407), anti-TfR (clone H68.4, Thermo Fisher, cat\# 136800) and anti-GAPDH (clone 6C5, Thermo Fisher, cat\# AM4300); rabbit polyclonal anti-GPP130 (Covance, cat\# AM4300) and anti-BiP (Affinity BioReagents, cat\# PA1-014); rat monoclonal anti-mouse IgM-allophycocyanin (APC) (clone RMM-1, BioLegend, cat\# 406509); goat polyclonal antimouse IgM-FITC (Thermo Fisher, cat\# 31992), anti-mouse IgG-AlexaFluor488 (Thermo Fisher, cat\# A11029), anti-rabbit IgG-AlexaFluor594 (Thermo Fisher, cat\# A11037) and anti-mouse IgG-phycoerythrin (PE) (BioLegend, cat\# 405307); and sheep polyclonal anti-mouse IgG-HRP (GE Healthcare, cat\# NA9310-1ML).

Plasmids. Primers used here are listed and numbered in Supplementary Table 1. To make the expression plasmid of PGAP4, we amplified hPGAP4 (TMEM246 or C9orf125) from cDNA library made from Hep3B by using primers No. 15 and 16. The amplified DNA was cut with EcoRI and NotI and cloned into pME-Zeo plasmid cut by the same enzymes. To construct the PGAP4-3HA plasmid, PGAP4 was amplified from pME-Zeo-hPGAP4 by primers No. 15 and 17. The amplified

Fig. 5 Functionally important residues in the UDP-GalNAc-binding cavity. a 3D structural model of PGAP4 with three TMDs in molecular surface display method. (Left) The whole structure of PGAP $4 \Delta \mathrm{N}$ with UDP-GalNAc. (Right) The model rotated by $90^{\circ}$ and cut to visualize the active site. Carbon, nitrogen, oxygen, sulfur, and hydrogen are shown in gray, blue, red, yellow, and white, respectively. $\mathbf{b}$ Enlarged view of PGAP4DN around UDP-GalNAc. Residues important for the activity are shown by sticks. c, d Western blotting (c) and immunofluorescence imaging (d) of various PGAP4 mutants. pMEhPGAP4-3HA-bearing WT or mutant sequences was transiently transfected into 3BT5-PGAP4-KO cells. c Representative data from two independent experiments. GAPDH, loading control. d GPP130, the Golgi marker. Scale bars: $10 \mu \mathrm{m}$. e Functional analysis of PGAP4 mutants. 3BT5-PGAP4-KO cells were transiently transfected with pME-hPGAP4-3HA for T334A mutant or pTK-hPGAP4-3HA harboring weak promoter for other mutants. Cells were stained with T5 mAb. Dotted lines, background staining. In lower panels, blue and red lines indicate PGAP4 WT and mutants, respectively. Quantitative data, mean fluorescence intensity $( \pm S D)$ from three independent experiments $(n=3)$. ${ }^{\star} p<0.05 ;{ }^{\star \star} p<0.005 ;{ }^{\star \star \star} p<0.0005$. e Statistical analyses were done by unpaired Student's t-test. See also Supplementary Fig. 5 and 9 
a

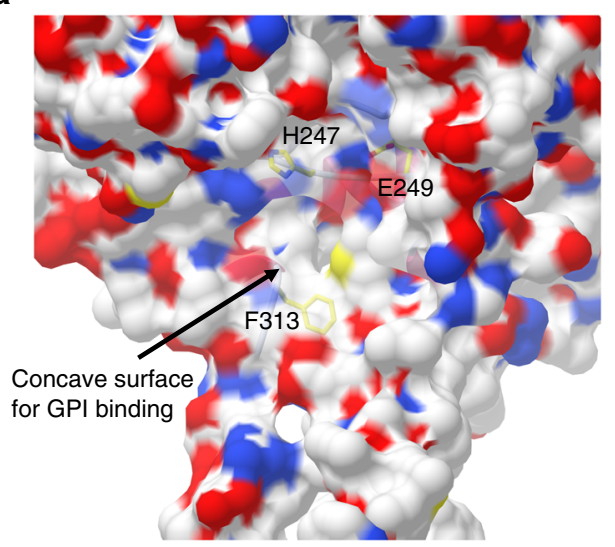

b

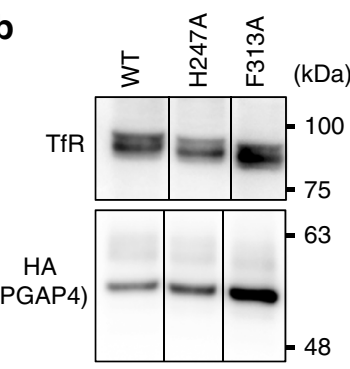

C

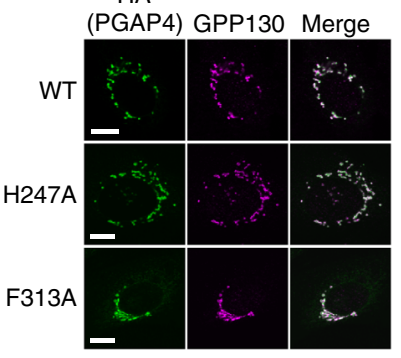

d --- W/o 1 st $A b$ - WT - Indicated genes 100 -

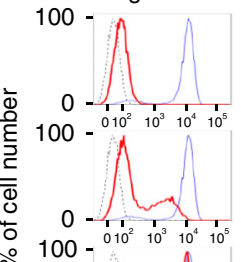
100

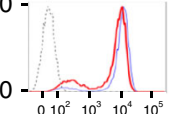

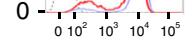

Fluorescence intensity of free GalNAc-GPI

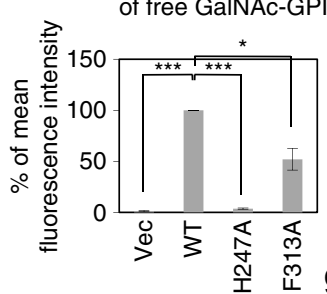

e

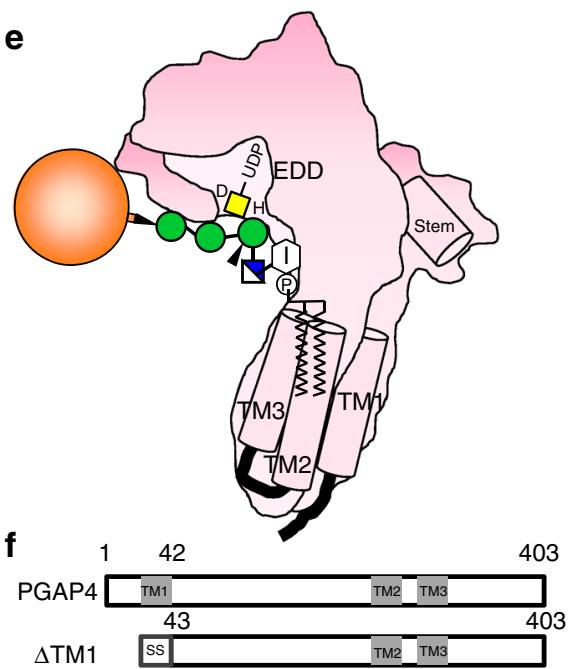

$\Delta \mathrm{TM} 1$

SS Signal sequence

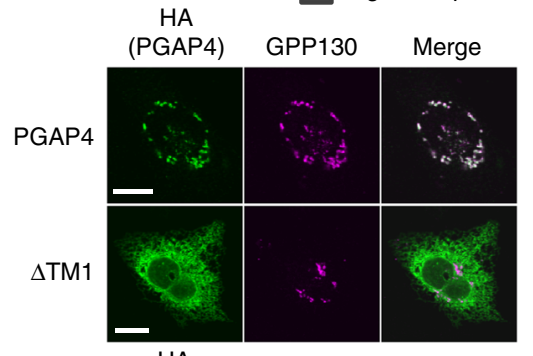

$\mathrm{HA}$ (PGAP4) BiP Merge
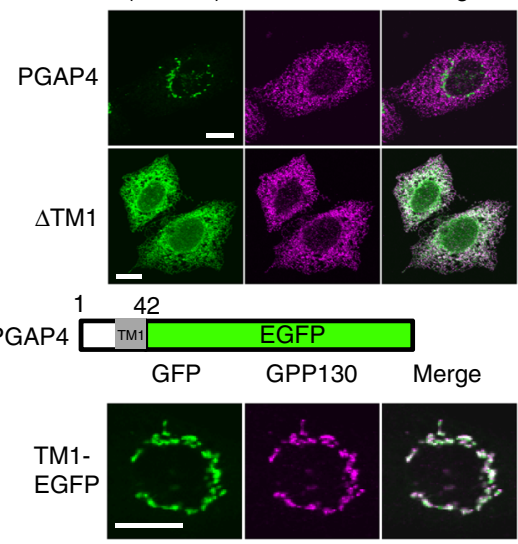

Fig. 6 Functional analysis of the concave surface in the juxtamembrane region and the first TMD. a Enlarged view of the concaving surface near the catalytic cavity. H247, E249, and F313 are exposed on the surface and located between UDP-GalNAc-binding site and TMDs. b-d Functional analysis of H247A and F313A mutant PGAP4. For western blotting (b) and immunofluorescence imaging (c), pME-hPGAP4-3HA with WT or mutant sequences was transiently transfected into 3BT5-PGAP4-KO cells. b TfR, loading control. c, d Representative data from two independent experiments. c GPP130, the Golgi marker. Scale bars: $10 \mu \mathrm{m}$. d Flow cytometry. 3BT5-PGAP4-KO cells were transiently transfected with pTK-hPGAP4-3HA. Cells were stained with T5 mAb. Dotted lines, background staining; blue lines, PGAP4 WT; red lines, PGAP4 mutants. Quantitative data of mean fluorescence intensity ( \pm SD) from three independent experiments $(n=3)$. Statistical analyses were done by unpaired Student's $t$-test. ${ }^{\star} p<0.05 ;{ }^{\star \star \star} p<0.0005$. e Schematic model for the interaction of PGAP4 with GPI-APs. PGAP4 exhibits a structure like a "golf club head with a short shaft" in which head portion corresponds to the catalytic domain. The catalytic domain harbors the cavity for UDP-GalNAc binding and the GPI-glycan binding concaving surface. The latter is closely located to the shaft corresponding to three bundled TMDs. The first mannose that accepts GalNAc (yellow square) should be presented close to the catalytic site (D363). GPI lipid might interact with TMDs. The space between the head portion and the membrane (not shown) may be sufficiently wide to accommodate entire GPI glycan. The protein part of various GPI-APs may be positioned out of this space not to interfere with the interaction with PGAP4. EDD, DXD-like motif; D, putative catalytic site D363; $\mathrm{H}, \mathrm{H} 247$ in GPI-binding region. $\mathbf{f}$ The localization of PGAP4 $\Delta T M 1$. (Upper) Schematic of the deletion mutant. Gray and white boxes indicated TMDs and signal sequence, respectively. (Lower) Immunofluorescence imaging of PGAP4DTM1. 3BT5-PGAP4KO cells were transiently transfected with pME-hPGAP4-3HA with WT or deletion mutant sequence. GPP130 and BiP, Golgi and ER markers, respectively. Scale bars: $10 \mu \mathrm{m}$. $\mathbf{g}$ Localization of TM1-EGFP. (Upper) Schematic of the chimera. (Lower) 3BT5-PGAP4-KO cells were transiently transfected with pMETM1-EGFP. GPP130, the Golgi marker. Scale bars: $10 \mu \mathrm{m}$. See also Supplementary Fig. 5 and 9 
sequence was cut with EcoRI and MluI followed by ligation into pME-3HA plasmid cut by the same enzymes. To construct pLIB2-Hyg-hPGAP4 and pLIB2-HyghPGAP4-3HA plasmid, pME-Zeo-hPGAP4 and pME-hPGAP4-3HA were digested by EcoRI and NotI followed by ligation with pLIB2-Hyg cut by the same enzymes. To make pME-3FLAG-hPGAP4-3HA plasmid, hPGAP4-3HA was amplified by using primers No. 18 and 19. The amplified sequence was cut with SalI and NotI, and ligated into pME-3FLAG plasmid cut by the same enzymes. To make pMEHyg-3FLAG-hPGAP4-3HA plasmid, 3FLAG-hPGAP4-3HA was cut with EcoRI and NotI followed by ligation with pME-Hyg plasmid cut by the same enzymes. To make PGAP4 $\triangle$ TM1 plasmid encoding 1-25 amino acids of hCD59 with 43-403 amino acids of hPGAP4, corresponding sequence was amplified from pME-
hPGAP4-3HA plasmid by using primers No. 20 and 21. The amplified sequence was inserted into pME plasmid with signal sequence of CD59, which was cut by PstI and NotI, by the In-Fusion Cloning Kit (TaKaRa). To make TM1-EGFP plasmid encoding 1-42 amino acids of hPGAP4 with enhanced green fluorescent protein (EGFP), corresponding sequence was amplified from pME-hPGAP4-3HA plasmid by using primers No. 15 and 22. The amplified sequence was cut with $E c o$ RI and MluI followed by ligation into pME-mEGFP plasmid cut by the same enzymes. To introduce mutation to plasmids, we followed the QuickChange SiteDirected Mutagenesis method. pME-hPGAP4-3HA was used as a template for the following mutants; N87A (primers No. 23 and 24), E211A (No. 29 and 30), D213A (No. 31 and 32), C43S (No. 35 and 36), C356S (No. 37 and 38), D363A (No. 39 and a

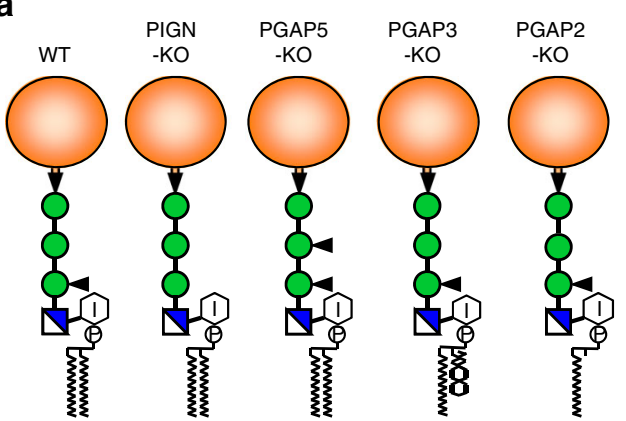

b
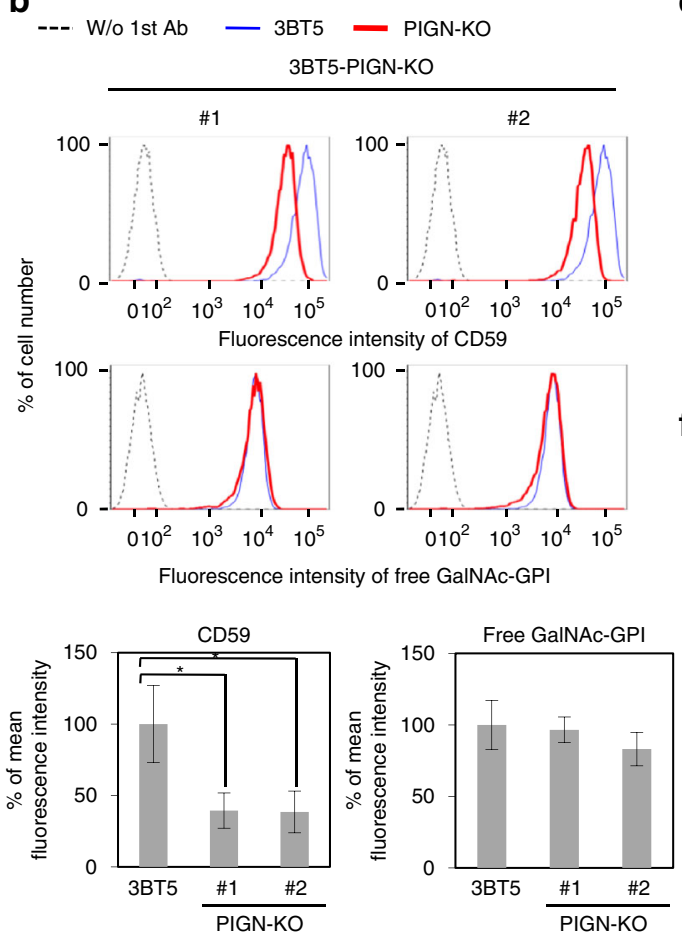

C C19-SLC35A2-KO

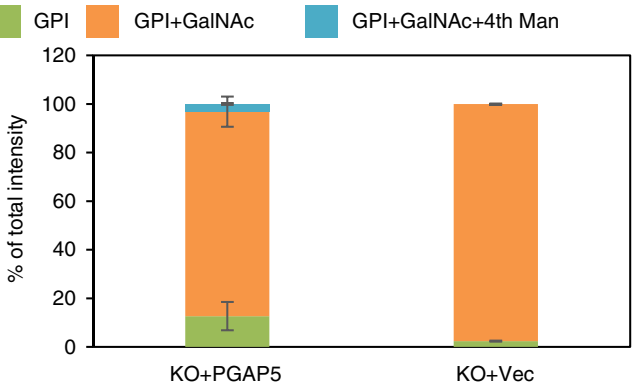

d

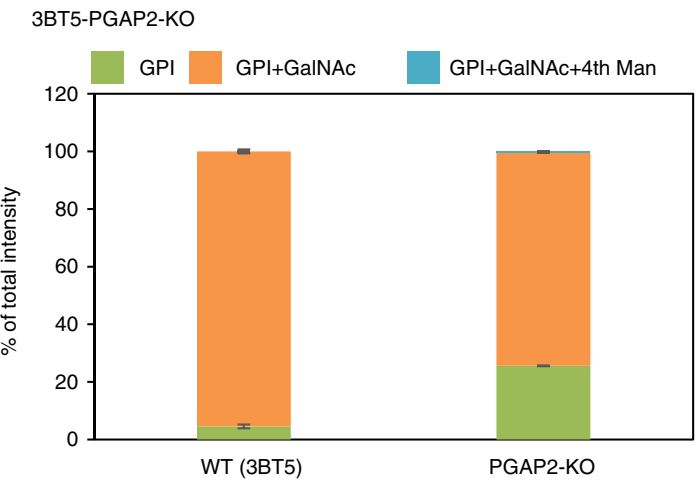

e

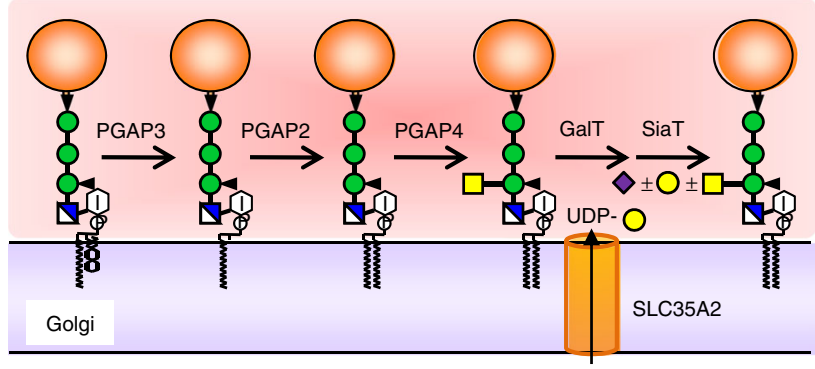

f
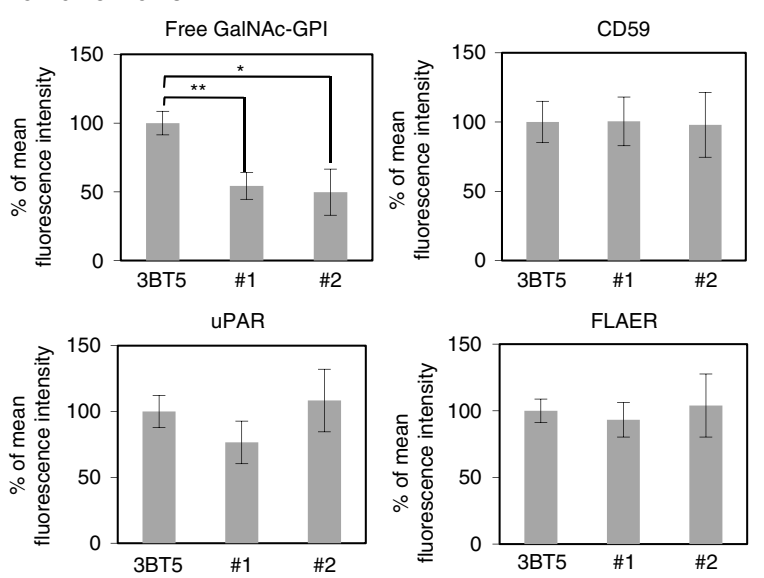

g

3BT5-PGAP3-KO

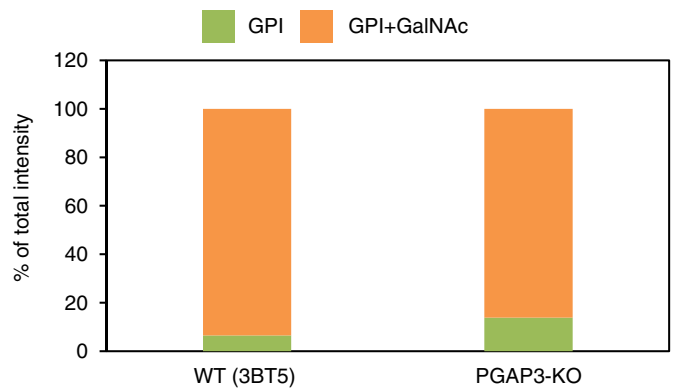

IDOI: 10.1038/s41467-017-02799-0 | www.nature.com/naturecommunications 
40), E249A (No. 41 and 42), H311A (No. 43 and 44), K362A (No. 45 and 46), M260A (No. 47 and 48), M270A (No. 49 and 50), M302A (No. 51 and 52), P335A (No. 53 and 54), R317A (No. 55 and 56), T334A (No. 57 and 58), V109A (No. 59 and 60), H247A (No. 61 and 62), and F313A (No. 63 and 64). pME-hPGAP4 (N87A)-3HA was used as a template for the following mutants; N87A, F283N (No. 25 and 26), and N87A, T347N (No. 27 and 28). pME-3FLAG-hPGAP4-3HA was used as a template for the mutant Q26K (Primers No. 33 and 34). pME-hPGAP4 (C43S)-3HA was used as a template for the double mutant C43S, C356S (No. 37 and 38). To construct pTK-hPGAP4-3HA, pME-hPGAP4-3HA was cut by SalI and $X b a \mathrm{I}$ and the obtained inserts were ligated with pTK-HA-hPIGY cut by XhoI and $\mathrm{XbaI}$. To make the Cas9 expression plasmids, pX330-EGFP was cut by BbsI followed by ligation with annealed primers designated as follows: hamPGAP4\#1 (primers No. 1 and 2), hamSLC35A2\#2 (No. 3 and 4), hamPIGN\#1 (No. 5 and 6), hamPIGN\#2 (No. 7 and 8), hamPGAP3\#1 (No. 9 and 10), hamPGAP3\#2 (No. 11 and 12), and hamPGAP2\#1 (No. 13 and 14). Sequences for sgRNAs were designed using CRISPRdirect ${ }^{42}$. The expression plasmid for hPIGN, hamPGAP5, and rPGAP2 were described previously and are available upon request ${ }^{3,6,43}$

Cell lines. CHO-K1 and Lec8 cells were obtained from ATCC. 3B2A cells were derived from CHO-K1 by stably expressing human DAF and CD5944. All cell lines used were cultured in Dulbecco's modified Eagle's medium/F-12 (Nacalai Tesque) supplemented with $10 \%$ fetal calf serum at $37^{\circ} \mathrm{C}$ under $5 \% \mathrm{v} / \mathrm{v} \mathrm{CO}$ condition. Cells except Lec8 cells were maintained in a medium supplemented with $600 \mu \mathrm{g} \mathrm{ml}^{-1}$ G418. Cells expressing His-FLAG-GST-FLAG-CD59 (HFGF-CD59) were cultured in a medium supplemented with $600 \mu \mathrm{g} \mathrm{ml}^{-1} \mathrm{G} 418$ and $8 \mu \mathrm{g} \mathrm{ml}{ }^{-1}$ blasticidin $\mathrm{S}$. Cells harboring empty vector or expression plasmids except HFGF-CD59 were cultured in a medium supplemented with $600 \mu \mathrm{g} \mathrm{ml}^{-1} \mathrm{G} 418,8 \mu \mathrm{g} \mathrm{ml}^{-1}$ blasticidin S, and $800 \mu \mathrm{g} \mathrm{ml}^{-1}$ hygromicin B.

To establish the $3 \mathrm{BT} 5$ cell line, T5-positive cells were enriched from wild-type 3B2A cells by three rounds of cell sorting, followed by limiting dilution. One clone, termed 3BT5, showed clear T5 staining without a T5 staining-negative population.

To make KO cell lines, pX330-EGFP plasmids harboring sgRNA of target genes were transiently transfected into cells by electroporation as described below. Three days after transfection, cells with EGFP fluorescence were collected by fluorescence-activated cell sorter (FACS) Aria II (BD), followed by limiting dilution to isolate $\mathrm{KO}$ clones. At least 1 week later, $\mathrm{KO}$ was validated.

To make stable transfectants except for 3BT5-PGAP4-KO cells expressing 3FLAG-hPGAP4(Q26K)-3HA, cells were infected with retrovirus harboring pLIB2-Hyg or pLIB2-BSD with relevant inserts in antibiotic-free medium as described below. To generate 3BT5-PGAP4-KO cells expressing 3FLAG-hPGAP4 (Q26K)-3HA, cells were electroporated with pME-Hyg-3FLAG-hPGAP4-3HA linearized by FspI. After 3 days culture post infection or transfection, cells were selected by the indicated antibiotics.

Plasmid transfection by electroporation. Cells $\left(5 \times 10^{6}\right)$ were suspended in $400 \mu \mathrm{l}$ of Opti-MEM and electroporated with $10 \mu \mathrm{g}$ of plasmid at $260 \mathrm{~V}$ and $1000 \mu \mathrm{F}$ using a Gene Pulser (Bio-Rad). Cells were then cultured for 3 days in antibiotic-free medium.

Infection of retrovirus. Cells receiving retrovirus were transiently transfected with pME-mCAT1 by electroporation. On the next day, culture medium was changed followed by further incubation for 2 days. PLAT-E packaging cells (Cell Biolabs) were seeded on 12 - or 6-well plates at $70 \%$ confluency. On the next day, cells were transfected with pLIB2-BSD or pLIB2-Hyg plasmid bearing CDNA of interest by using PEImax transfection reagent (Polysciences, 24765-2). After about $10 \mathrm{~h} \mathrm{cul-}$ ture, $1 \mathrm{M}$ sodium butyrate was added to $10 \mathrm{mM}$ final concentration and further incubated for $12 \mathrm{~h}$. On the next day, medium was replaced by a fresh one and incubated at $32^{\circ} \mathrm{C}$ for $24 \mathrm{~h}$. Culture medium was added to cells expressing mCAT1, and these cells were cultured at $32^{\circ} \mathrm{C}$ for $24 \mathrm{~h}$. On the next day, culture medium was changed, and cells were cultured at $37^{\circ} \mathrm{C}$.
Gene-trapping screening. pCMT-SApA-BSD plasmid that contains a splice acceptor sequence for gene-trapping was constructed previously ${ }^{22}$. As recipient cells, 3BT5 cells in one 15-cm dish were transfected with pME-mCAT1 plasmid by electroporation followed by incubation at $37^{\circ} \mathrm{C}$ in eight 6-well plates for 3 days. For virus production, PLAT-E cells in two $15-\mathrm{cm}$ dishes were transfected with pCMTSApA-BSD plasmid using calcium phosphate transfection method. Twelve hours later, $1 \mathrm{M}$ sodium butyrate was added to $10 \mathrm{mM}$ final concentration. Twelve hours later, medium was removed and fresh medium was added followed by incubation at $32^{\circ} \mathrm{C}$. Twenty four hours later, supernatant containing virus was mixed with 8 $\mu \mathrm{g} \mathrm{ml}^{-1}$ polybrene. $3 \mathrm{BT} 5$ cells expressing mCAT1 were infected with the prepared virus solution by centrifugation at $1220 \times g$ for $2 \mathrm{~h}$ at $32^{\circ} \mathrm{C}$. From 2 days after infection, selection was performed with $8 \mu \mathrm{g} \mathrm{ml}^{-1}$ blasticidin $\mathrm{S}$ for 1 week. Mutant cells with negative staining with T5 antibody (1:100 ascites) were collected twice by cell sorting using FACS Aria II to enrich mutant cells defective in GPI-GalNAc biosynthesis. For third round of sorting, mutant cells were double stained with T5 and anti-CD59 $\left(10 \mu \mathrm{g} \mathrm{ml}^{-1}\right)$ antibodies, and we collected cells with CD59-positive (GPI-AP-positive) but T5-negative staining to eliminate cells defective in GPIanchor biosynthesis. Cells with CD59 and T5 double-negative staining were also collected for control experiments.

Next-generation sequencing. Genomic DNA was prepared from $2 \times 10^{7}$ cells using the Wizard Genomic DNA Purification Kit (Promega) according to the manufacturer's protocol. Genomic DNA $(15 \mu \mathrm{g})$ was fragmented by Covaris S2 (M\&S Instruments Inc.) according to 800 bp protocol followed by repairing by T4 DNA polymerase (NEB) and phosphorylation by T4 PNK (NEB). Splinkerette adaptors (No. 65 and 66, see Supplementary Table 1) ${ }^{45}$ were ligated. After column purification, the fragments were used for templates of splinkerette PCR using primers No. 67 and 68. The resulting DNA fragments were further amplified by nested PCRs using primers No. 69 and 70, followed by primers No. 71 and 72 . Illumina P5 and P7 sequences and barcode sequences were attached to the products by 4 cycles of PCR with $100 \mathrm{ng}$ each of initial PCR product as the template. Paired-end sequencing (101-bp $\times 2$ ) was performed on the HiSeq 2500 (Illumina) platform. The numbers of reads obtained from control and S3 cells were approximately 15 million and 9 million, respectively.

Analysis of gene-trap insertions. In order to map reads derived from nextgeneration sequencing described in the preceding section, we used the genome assembly from the CHO cell line CHO-K1 (Accession: GCF_000223135.1, Beijing Genomic Institute $)^{46}$ as templates. Because the genomic assembly of $\mathrm{CHO}$ cells was incomplete and the entire genomic sequences were assembled in 0.1 million scaffolds, we discarded scaffolds $<1 \mathrm{Mbp}$ and used only 641 long scaffolds as mapping templates. FASTQ data files were analyzed by the CLC Genomic Workbench software version 7.0.4 (QIAGEN) ${ }^{21}$,22. In brief, after quality trimming and removal of the common LTR sequence, the 50 base pair reads were mapped onto 641 selected scaffolds. To avoid duplicate mapping, duplicate reads were removed. RPKM score of each scaffold was calculated and ranked. The regions highly mapped by the reads were picked up and the sequences were analyzed by Basic Local Alignment Search Tool (BLAST)

Flow cytometry. To detect free GalNAc-GPI, cells were treated with or without final $2 \mathrm{mg} \mathrm{ml}^{-1}$ pronase in Opti-MEM containing $1 \mathrm{mM} \mathrm{CaCl}$ at $37^{\circ} \mathrm{C}$ for $2 \mathrm{~h}$. Cells were stained with mouse monoclonal anti-free GalNAc-GPI (T5-4E10) $(1: 100$ ascites) in FACS buffer (phosphate-buffered saline (PBS) containing 1\% bovine serum albumin (BSA) and $0.1 \% \mathrm{NaN}_{3}$ ) followed by rat monoclonal anti-mouse immunoglobulin M (IgM) conjugated with APC or goat polyclonal mouse antiIgM conjugated with FITC. To detect GPI-APs, anti-CD59 $\left(10 \mu \mathrm{g} \mathrm{ml}^{-1}\right)$ and anti$\operatorname{uPAR}\left(10 \mu \mathrm{g} \mathrm{m}^{-1}\right)$ followed by polyclonal goat anti-mouse IgG conjugated with PE (1:100). Cells were also stained with FLAER (Cedarlane Laboratories, $1: 100$ ), which is a FITC-conjugated non-toxic bacterial toxin aerolysin specifically binding to GPI-APs. For lectin staining, lectins were diluted (1:200) in FACS buffer containing

Fig. 7 Acceptor substrate specificity, timing of GalNAc modification, and T5 mAb epitope characteristics. a Schematic of the structure of GPI-APs in various mutant cells. b T5 mAb staining of PIGN-KO cells. (Upper) Surface expression of CD59 and the staining with T5 mAb analyzed by FACS. Dotted lines, background staining; blue lines, 3BT5 cells; red lines, PIGN-KO cells. (Lower) Mean fluorescence intensity ( \pm SD) from three independent experiments. Statistical analyses were done by unpaired Student's $t$-test. ${ }^{*} p<0.05$. \#1 and \#2, clones generated by two different sgRNAs. c Percentage of GalNAc modification of CD59-GPI in PGAP5 mutant (C19) cells. C19-SLC35A2-KO cells were transiently transfected with empty vector or pME-hyghamPGAP5. Percentage of total intensity (mean \pm SD) was calculated from the peak areas obtained by two independent measurements with LC-ESI-MS/ MS. d Percentage of GalNAc modification of CD59-GPI in PGAP2-KO cells. e The proposed biosynthetic pathway of GPI-APs in the Golgi. Fatty acid remodeling by PGAP3 and PGAP2 and then GalNAc side-chain modification by PGAP4 occur. GalNAc residue can be further elongated by galactose (Gal) and sialic acid (Sia). GalT and SiaT for GPI modification are not identified yet. UDP-Gal is supplied by SLC35A2. f Flow cytometry of PGAP3-KO cells. Staining for T5, CD59, uPAR, and FLAER. Mean fluorescence intensity $( \pm$ SD) from three independent experiments. Statistical analyses were done by unpaired Student's t-test. ${ }^{\star} p<0.05 ;{ }^{\star *} p<0.005$. \#1 and \#2, clones generated by two different sgRNAs. $\mathbf{g}$ Percentage of GalNAc modification of CD59-GPI in PGAP3-KO cells. See also Supplementary Fig. 3, 10, 11 and 12 
$1 \mathrm{mM} \mathrm{CaCl} 2, \mathrm{MgCl}_{2}$, and $\mathrm{MnCl}_{2}$. The data were collected by FACS Canto II (BD) and analyzed by the FlowJo software.

Genotyping of 3BT5 cell line. mRNA of 3BT5 was prepared from $1 \times 10^{6}$ cells by the RNeasy Mini Kit (QIAGEN). A sample of cDNA was prepared by reverse transcription reaction using SuperScript VILO Master Mix (Thermo Fisher). SLC35A2 was amplified by specific primers (No. 73 and 74) followed by Sanger sequencing.

DRM separation. Cells were lysed in lysis-buffer A containing $50 \mathrm{mM}$ Tris- $\mathrm{HCl}$ (pH 7.4), $150 \mathrm{mM} \mathrm{NaCl}, 1 \%$ Triton X-100, $5 \mathrm{mM}$ EDTA, and $1 \times$ protease inhibitor cocktail followed by centrifugation at $21,900 \times g$ for $15 \mathrm{~min}$ at $4^{\circ} \mathrm{C}$. Supernatants were collected as Triton-soluble fraction (S). Pellets containing DRM fraction were suspended by lysis-buffer B containing $50 \mathrm{mM}$ Tris- $\mathrm{HCl}(\mathrm{pH} 7.4), 150 \mathrm{mM} \mathrm{NaCl}$, $60 \mathrm{mM}$ Octyl- $\beta$-D-glucoside, $1 \mathrm{mM}$ EDTA, and $1 \times$ protease inhibitor cocktail followed by centrifugation at the same condition. Supernatants were collected as Triton-resistant fraction (R). Each of S or R samples was mixed by opposite buffer to set same buffer conditions. $4 \times$ sodium dodecyl sulfate (SDS) sample buffer without $\beta$-mercaptoethanol was added followed by boiling at $95^{\circ} \mathrm{C}$ for $5 \mathrm{~min}$. Samples were then analyzed by western blotting.

Western blotting. For the PGAP4 expression analysis, cell lysates were prepared by lysis-buffer $\mathrm{A}$. Lysates were centrifuged at $21,900 \times g$ for $15 \mathrm{~min}$ at $4{ }^{\circ} \mathrm{C}$ Supernatant was recovered and mixed with $4 \times$ SDS-sample buffer with $5 \% \beta$ mercaptoethanol followed by incubation on ice for $30 \mathrm{~min}$. Samples were run on SDS-polyacrylamide gel electrophoresis (PAGE) gels and transferred to polyvinylidene difluoride membranes. Antibodies used were mouse monoclonal antiHA (1:2000), anti-TfR (1:1000), anti-GAPDH (1:4000), anti-CD59 $\left(0.5 \mu \mathrm{g} \mathrm{ml}^{-1}\right)$, anti-DAF $\left(0.5 \mu \mathrm{g} \mathrm{ml}^{-1}\right)$, anti-uPAR $\left(0.5 \mu \mathrm{g} \mathrm{m}^{-1}\right)$, and anti-Caveolin 1 (1:1000). Relevant areas in blots are shown in figures and scans of uncropped blots are shown in Supplementary Fig. 13.

Protein purification. For purification of HFGF-CD59 6 , $2 \times 10^{8}$ cells stably expressing HFGF-CD59 were treated with 1 unit $\mathrm{ml}^{-1}$ PI-PLC in $10 \mathrm{ml}$ of PI-PLC buffer (Opti-MEM containing $10 \mathrm{mM}$ HEPES-NaOH (pH 7.4), $1 \mathrm{mM}$ EDTA and $0.1 \% \mathrm{BSA}$ ) at $37^{\circ} \mathrm{C}$ for $2 \mathrm{~h}$. Supernatant was loaded to a column of $500 \mu \mathrm{l}$ of Glutathione Sepharose $4 \mathrm{~B}$ at $4{ }^{\circ} \mathrm{C}$. After washing with $10 \mathrm{ml}$ PBS, HFGF-CD59 was eluted by elution-buffer A (pH7.4, PBS containing $30 \mathrm{mM}$ HEPES-NaOH (pH 7.4) and $20 \mathrm{mM}$ reduced glutathione). Trichloroacetic acid (TCA; Wako) was added to eluted samples to final $10 \%$ followed by incubation on ice for $30 \mathrm{~min}$. Proteins were precipitated by centrifugation at $13,400 \times g$ for $30 \mathrm{~min}$ at $4{ }^{\circ} \mathrm{C}$. After twice washing with $100 \%$ ice-cold ethanol, pellets were resolved in $1 \times$ SDS-sample buffer with $5 \%$ $\beta$-mercaptoethanol and boiled at $95^{\circ} \mathrm{C}$ for $5 \mathrm{~min}$. Samples were run on SDS-PAGE gel and stained by Imperial Protein Stain. For PGAP2-KO cells, $200 \mathrm{ml}$ culture medium from ten $15-\mathrm{cm}$ dishes were loaded to a column of $500 \mu \mathrm{l}$ of Glutathione Sepharose $4 \mathrm{~B}$ followed by the same procedures described above.

To determine the disulfide bonds of PGAP4, $2 \times 10^{8}$ cells stably expressing 3FLAG-hPGAP4(Q26K)-3HA were lysed in lysis-buffer A. After centrifugation at $13,400 \times g$ for $15 \mathrm{~min}$ at $4{ }^{\circ} \mathrm{C}$, supernatant was loaded to a column of $500 \mu \mathrm{l}$ of FLAG-M2 beads (Sigma-Aldrich) at $4{ }^{\circ} \mathrm{C}$. After washing with $10 \mathrm{ml}$ PBS containing 1\% Triton X-100, 3FLAG-hPGAP4(Q26K)-3HA was eluted by elution-buffer B (PBS containing $20 \mathrm{mM}$ HEPES (pH 7.4) and $150 \mu \mathrm{g} \mathrm{m}^{-1} 3 \times$ FLAG peptide). Eluted samples were precipitated by TCA. Pellet was resolved in $1 \times$ SDS-sample buffer without $\beta$-mercaptoethanol to keep disulfide bridges. Samples were run on SDS-PAGE gel and stained by Imperial Protein Stain.

Mass spectrometry. To analyze the GPI-containing peptides from HFGF-CD59, protein bands were excised and reduced with $10 \mathrm{~mm}$ dithiothreitol (DTT), followed by alkylation with $55 \mathrm{~mm}$ iodoacetamide, and digested in-gel by treatment with trypsin. The resultant peptides were subjected to mass spectrometric analyses by MALDI-TOF or LC-ESI system. MALDI-TOF MS analysis was performed by AXIMA Resonance (Shimadzu/Kratos) in the positive ion mode. The system was controlled by the Shimadzu Biotech MALDI-MS software. Ionization was performed with $337 \mathrm{~nm}$ pulsed $\mathrm{N}_{2}$ laser. Helium and argon gases were used for ion cooling and collision-induced dissociation, respectively. For sample preparation, 1 $\mu \mathrm{l}$ aliquots of the peptides were purified with Zip-Tip ${ }_{\mu-\mathrm{Cl}}$ pipette tips (Millipore) and deposited directly in matrix $\left(0.5 \mu \mathrm{l}\right.$ of $5 \mathrm{mg} \mathrm{m}^{-1} 2,5$-dihydroxybenzoic acid (Shimadzu GLC) in 50\% acetonitrile containing $0.05 \%$ trifluoroacetic acid) onto the MALDI target plate. The solution on the target plate was completely dried, and then the plate was introduced into the instrument. The instrument was calibrated using an external standard peptide mixture of human ACTH peptide fragment 18-39 $\left([\mathrm{M}+\mathrm{H}]^{+}, 2465.20\right)$ and bovine insulin oxidized B chain $\left([\mathrm{M}+\mathrm{H}]^{+}\right.$, 3494.65). For quantification, data were obtained by nanocapillary reversed-phase LC-MS/MS using a C18 column $(0.1 \times 150 \mathrm{~mm})$ on a nanoLC system (Advance, Michrom BioResources) coupled to an LTQ Orbitrap Velos mass spectrometer (Thermo Fisher). The mobile phase consisted of water containing $0.1 \%$ formic acid (solvent A) and acetonitrile (solvent B). Peptides were eluted by a gradient of

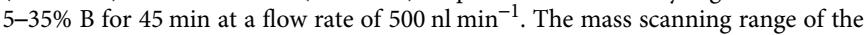
instrument was set at $m / z 350-1500$. The ion spray voltage was set at $1.8 \mathrm{kV}$ in the positive ion mode. The MS/MS spectra were acquired by automatic switching between MS and MS/MS modes (with collisional energy set to 35\%). Helium gas was used as collision gas.

Xcalibur Software (Thermo Fisher) was used for analysis of mass data. In the MS/MS profiles, those that contain characteristic fragments derived from GPI anchors, such as fragment ions of $m / z 422^{+}$and $447^{+}$, were selected, and fragments in the selected profiles were assigned to determine the GPI structures. Based on the profiles of the MS/MS fragments, the peak areas of the parental MS fragments corresponding to predicted GPI-peptides (Supplementary Table 2, 3, 4, 5) were measured and the ratio in the total GPI peptide fragments was calculated.

To determine the disulfide bridges in PGAP4, bands were excised after SDSPAGE under non-reducing conditions and were subjected to in-gel digestion by chymotrypsin either without or with reduction and alkylation with $10 \mathrm{~mm}$ DTT and $55 \mathrm{~mm}$ iodoacetamide (+DTT). Peptides from the digested samples were analyzed by nanoLC-MS/MS. Peptides corresponding to PGAP4 were identified by database searching in-house MASCOT Server (Matrix Science). Precursor mass tolerance was set to $10 \mathrm{ppm}$ and $0.8 \mathrm{Da}$ for Orbitrap and linear ion trap, respectively. Dehydrogenation of cysteine and oxidization of methionine were set as dynamic modification. Disulfide-linked peptide adducts were identified using an algorithm DBond developed by Choi et al. ${ }^{47}$ (Supplementary Fig. $7 \mathrm{~b}$ and $\mathrm{c}$ and Supplementary Table 6).

Immunofluorescence imaging. The cells were fixed with PBS containing 4\% paraformaldehyde for $20 \mathrm{~min}$ at room temperature, followed by washing with 40 $\mathrm{mM}$ glycine ( $\mathrm{pH} 7.4$ ) for $10 \mathrm{~min}$. After permeabilization with $0.1 \%$ saponin or Triton X-100, cells were double stained with mouse anti-HA (1:100) and rabbit anti-GPP130 (1:200) or anti-BiP (1:100) antibody followed by Alexa 488conjugated goat anti-mouse IgG (1:1000) and Alexa 594-conjugated goat rabbitmouse IgG (1:1000). Confocal images were acquired on a FluoView FV1000 (Olympus)

In silico analysis. PGAP4 transmembrane segments were determined using

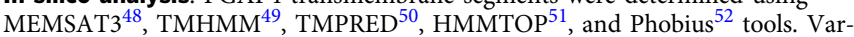
ious protein topology prediction tools predicted first TMD (TM1) to be located at the beginning of N-terminus and two consecutive TMDs (TM2 and TM3) are located in the middle of the sequence. A consensus from all the prediction tools was considered and locations of TM1, TM2, and TM3 are predicted to be W20A42, I262-A284, and S289-V308, respectively. The BLAST tool ${ }^{53}$ was used to align PGAP4 sequences against those present in PDB $^{54}$. Levels of PGAP4 mRNA in various tissues were analyzed by BioGPS ${ }^{23}$

Comparative modeling of PGAP4 structure. Owing to a lack of sufficient sequence similarity with any known protein in the PDB data bank, homology modeling of the PGAP4 was not straightforward. We first modeled an initial structure of the PGAP4 $\Delta$ TM2, TM3 $(\Delta 262-308)$ using a hierarchical protein structure modeling approach of server I-TASSER ${ }^{28}$. I-TASSER successfully identified structural templates from the PDB by multiple threading approach LOMETS $^{55}$ and constructed an atomic model of PGAP4ATM2, TM3 by iterative template fragment assembly simulations. We selected the top scoring atomic model for analysis. The overall quality of selected homology model analyzed by PROSA$\mathrm{Web}^{29}$ shows a poor score (score $=-4.89$ ) but it is still within the range of lowresolution experimental structures of this size of 356 residues. The Ramachandran plot analysis of the backbone residues generated by RAMPAGE ${ }^{30}$ shows $71.5 \%$ residues in the favored region, $17.2 \%$ in the allowed region, and $11.3 \%$ in the outlier region. Thus our structure model satisfies the quality considering its rather large size, which could be a limitation for modeling. Three disulfide bridges (C132-C136, C144-C194, and C332-C333) confirmed by LC-ESI-MS/MS analysis were feasible in this model. The distances between $\mathrm{C} \alpha$ carbons of C132-C136, C144-C194, and C332-C333 pairs were $10.2 \AA, 8.9 \AA$, and $3.8 \AA$, respectively. The presence of a disulfide bridge $\mathrm{C} 144-\mathrm{C} 194$ in the PGAP4 provided a solid basis to validate overall topology of the GT-A fold in modeled structure. Out of the top 10 templates selected from the LOMETS threading programs, the structure of bacterial cellulose synthase $^{27}$ (PDB ID: 4P02) showed sufficient structural similarity (Template Modeling score $=0.813$ ).

Based on this sequence and structural similarity information from the ITASSER server, we built a model of PGAP4 $\triangle \mathrm{N}$ using homology modeling approach in MODELLER9v1 $7^{31}$. Since templates identified by the meta-server threading approach implemented in LOMETS (integrated with I-TASSER) finds some significant threading alignment with PDB library, we used X-ray structure of cellulose synthase and fold recognition model of GT-A fold (template structures) together with sequence alignment between PGAP4 and cellulose synthase GT-A fold region obtained from pGenThreader ${ }^{56}$ to model PGAP $4 \Delta \mathrm{N}$ structure. Since there was not enough structural similarity of transmembrane regions with transmembrane region of PGAP4 or any other structure in protein databank, we align TM region residues to gaps in the template sequences (Supplementary Fig. 6a, b). We further used secondary structure information and restrains to model the structure and orientation of TMDs.

The secondary structure information predicted by program PSIPREDv $3.3^{57}$ was used to restrain residues to achieve proper $\alpha$ helice and $\beta$ sheet structures. It is 
achieved by special restraints on residues W20-L41, R45-F51, L53-N59, Q63-E81, H116-Q131, D190-S197, Q219-F232, M260-R282, W291-L307, A344-S352, and K362-A372 to form $\alpha$ helices and residues I104-V109, Y206-M209 to be $\beta$ sheets. Additionally, restrains to form disulfide bridges between C132-C136, C144-C194, and C332-C333 pairs were introduced. The secondary structure of TMDs was set to $\alpha$ helix through restraints. The backbone carbon atoms of W20-F283, N63-Y117, E82-V326, A39-Y119, and L53-D110 residues were harmonic restrained to attain a position within $10 \AA$ from each other.

Finally, MODELLER was run using the slow level of VTFM optimization repeated twice for 300 iterations. This thorough setup is slower and timeconsuming but expected to result in more accurate model construction. Regions that were not covered by alignment were modeled by loop modeling procedure in MODELLER. The DOPE (Discrete Optimized Protein Energy) ${ }^{58}$ and GA341 scores ${ }^{59}$ were calculated for each model and the top 10 models were analyzed visually. Top scoring model without any knot was selected as a final model and discussed here. The overall quality of final model analyzed by RAMPAGE shows $88.2 \%$ residues in the favored region, $5.5 \%$ in the allowed region and $6.3 \%$ in the outlier region.

Quantification and statistical analysis. Statistical analyses were done using Microsoft Excel. To compare between two individual groups, unpaired Student's $t$-test was used. The number of repeats of each experiment is indicated at each figure legend. All quantitative data presented here are means $\pm \mathrm{SD}$. We considered $p$-values $<0.05$ as statistically significant

Data availability. The mass spectrometric proteomics data have been deposited to the ProteomeXchange Consortium via the PRIDE ${ }^{60}$ partner repository with the dataset identifiers PXD008137 (project name "Determination of disulfide bridges of human PGAP4 protein") and PXD008230 (project name "Determination of GPI structure of human $\mathrm{CD} 59$ in various $\mathrm{KO} \mathrm{CHO}$ cells"). All other data supporting the findings are available from the corresponding author.

Received: 27 July 2017 Accepted: 28 December 2017

Published online: 26 January 2018

\section{References}

1. Kinoshita, T. \& Fujita, M. Biosynthesis of GPI-anchored proteins: special emphasis on GPI lipid remodeling. J. Lipid Res. 57, 6-24 (2016).

2. Tanaka, S., Maeda, Y., Tashima, Y. \& Kinoshita, T. Inositol deacylation of glycosylphosphatidylinositol-anchored proteins is mediated by mammalian PGAP1 and yeast Bst1p. J. Biol. Chem. 279, 14256-14263 (2004).

3. Fujita, M. et al. GPI glycan remodeling by PGAP5 regulates transport of GPIanchored proteins from the ER to the Golgi. Cell 139, 352-365 (2009).

4. Fujita, M. et al. Sorting of GPI-anchored proteins into ER exit sites by p24 proteins is dependent on remodeled GPI. J. Cell Biol. 194, 61-75 (2011).

5. Maeda, Y. et al. Fatty acid remodeling of GPI-anchored proteins is required for their raft association. Mol. Biol. Cell 18, 1497-1506 (2007).

6. Tashima, Y. et al. PGAP2 is essential for correct processing and stable expression of GPI-anchored proteins. Mol. Biol. Cell 17, 1410-1420 (2006).

7. Kinoshita, T. Biosynthesis and deficiencies of glycosylphosphatidylinositol. Proc. Jpn. Acad. Ser. B Phys. Biol. Sci. 90, 130-143 (2014).

8. Grimme, S. J., Westfall, B. A., Wiedman, J. M., Taron, C. H. \& Orlean, P. The essential Smp3 protein is required for addition of the side-branching fourth mannose during assembly of yeast glycosylphosphatidylinositols. J. Biol. Chem. 276, 27731-27739 (2001).

9. Taron, B. W., Colussi, P. A., Wiedman, J. M., Orlean, P. \& Taron, C. H. Human Smp3p adds a fourth mannose to yeast and human glycosylphosphatidylinositol precursors in vivo. J. Biol. Chem. 279, 36083-36092 (2004).

10. Homans, S. W. et al. Complete structure of the glycosyl phosphatidylinositol membrane anchor of rat brain Thy-1 glycoprotein. Nature 333, 269-272 (1988).

11. Stahl, N. et al. Glycosylinositol phospholipid anchors of the scrapie and cellular prion proteins contain sialic acid. Biochemistry 31, 5043-5053 (1992).

12. Bate, C., Nolan, W. \& Williams, A. Sialic acid on the glycosylphosphatidylinositol anchor regulates PrP-mediated cell signaling and prion formation. J. Biol. Chem. 291, 160-170 (2016).

13. Stanley, P. What have we learned from glycosyltransferase knockouts in mice? J. Mol. Biol. 428, 3166-3182 (2016).

14. Albesa-Jove, D. \& Guerin, M. E. The conformational plasticity of glycosyltransferases. Curr. Opin. Struct. Biol. 40, 23-32 (2016).

15. Kellokumpu, S., Hassinen, A. \& Glumoff, T. Glycosyltransferase complexes in eukaryotes: long-known, prevalent but still unrecognized. Cell. Mol. Life. Sci. 73, 305-325 (2016)

16. Wiggins, C. A. \& Munro, S. Activity of the yeast MNN1 alpha-1,3mannosyltransferase requires a motif conserved in many other families of glycosyltransferases. Proc. Natl Acad. Sci. USA 95, 7945-7950 (1998).
17. Tomavo, S. et al. Immunolocalization and characterization of the low molecular weight antigen (4-5 kDa) of Toxoplasma gondii that elicits an early IgM response upon primary infection. Parasitology 108, 139-145 (1994). Pt 2.

18. Striepen, B. et al. Molecular structure of the "low molecular weight antigen" of Toxoplasma gondii: a glucose alpha 1-4 N-acetylgalactosamine makes free glycosyl-phosphatidylinositols highly immunogenic. J. Mol. Biol. 266, 797-813 (1997).

19. Kang, J. Y. et al. PIG-V involved in transferring the second mannose in glycosylphosphatidylinositol. J. Biol. Chem. 280, 9489-9497 (2005).

20. Stanley, P. Selection of specific wheat germ agglutinin-resistant (WgaR) phenotypes from Chinese hamster ovary cell populations containing numerous lecR genotypes. Mol. Cell. Biol. 1, 687-696 (1981).

21. Carette, J. E. et al. Ebola virus entry requires the cholesterol transporter Niemann-Pick C1. Nature 477, 340-343 (2011).

22. Hirata, T. et al. Post-Golgi anterograde transport requires GARP-dependent endosome-to-TGN retrograde transport. Mol. Biol. Cell 26, 3071-3084 (2015).

23. Wu, C. et al. BioGPS: an extensible and customizable portal for querying and organizing gene annotation resources. Genome Biol. 10, R130 (2009).

24. Cong, L. et al. Multiplex genome engineering using CRISPR/Cas systems. Science 339, 819-823 (2013).

25. Mali, P. et al. RNA-guided human genome engineering via Cas9. Science 339, 823-826 (2013)

26. Nakano, Y., Noda, K., Endo, T., Kobata, A. \& Tomita, M. Structural study on the glycosyl-phosphatidylinositol anchor and the asparagine-linked sugar chain of a soluble form of CD59 in human urine. Arch. Biochem. Biophys. 311, 117-126 (1994).

27. Morgan, J. L., McNamara, J. T. \& Zimmer, J. Mechanism of activation of bacterial cellulose synthase by cyclic di-GMP. Nat. Struct. Mol. Biol. 21, 489-496 (2014).

28. Yang, J. et al. The I-TASSER Suite: protein structure and function prediction. Nat. Methods 12, 7-8 (2015).

29. Wiederstein, M. \& Sippl, M. J. ProSA-web: interactive web service for the recognition of errors in three-dimensional structures of proteins. Nucleic Acids Res. 35, W407-W410 (2007).

30. Lovell, S. C. et al. Structure validation by Calpha geometry: phi,psi and Cbeta deviation. Proteins 50, 437-450 (2003).

31. Eswar, N. et al. Comparative protein structure modeling using MODELLER. Curr. Protoc. Protein Sci. Chapter 2, Unit 2.9 (2007).

32. Opat, A. S., van Vliet, C. \& Gleeson, P. A. Trafficking and localisation of resident Golgi glycosylation enzymes. Biochimie 83, 763-773 (2001).

33. Hong, Y. et al. Pig-n, a mammalian homologue of yeast Mcd4p, is involved in transferring phosphoethanolamine to the first mannose of the glycosylphosphatidylinositol. J. Biol. Chem. 274, 35099-35106 (1999).

34. Almeida, I. C. et al. Highly purified glycosylphosphatidylinositols from Trypanosoma cruzi are potent proinflammatory agents. EMBO J. 19, 1476-1485 (2000).

35. Gill, D. J., Clausen, H. \& Bard, F. Location, location, location: new insights into O-GalNAc protein glycosylation. Trends Cell. Biol. 21, 149-158 (2011).

36. Kimmel, J. et al. Membrane topology and transient acylation of Toxoplasma gondii glycosylphosphatidylinositols. Eukaryot. Cell 5, 1420-1429 (2006).

37. Mukasa, R., Umeda, M., Endo, T., Kobata, A. \& Inoue, K. Characterization of glycosylphosphatidylinositol (GPI)-anchored NCAM on mouse skeletal muscle cell line $\mathrm{C} 2 \mathrm{C} 12$ : the structure of the GPI glycan and release during myogenesis. Arch. Biochem. Biophys. 318, 182-190 (1995).

38. Brewis, I. A., Ferguson, M. A., Mehlert, A., Turner, A. J. \& Hooper, N. M. Structures of the glycosyl-phosphatidylinositol anchors of porcine and human renal membrane dipeptidase. Comprehensive structural studies on the porcine anchor and interspecies comparison of the glycan core structures. J. Biol. Chem. 270, 22946-22956 (1995).

39. Deeg, M. A. et al. Glycan components in the glycoinositol phospholipid anchor of human erythrocyte acetylcholinesterase. Novel fragments produced by trifluoroacetic acid. J. Biol. Chem. 267, 18573-18580 (1992).

40. Roberts, W. L., Santikarn, S., Reinhold, V. N. \& Rosenberry, T. L. Structural characterization of the glycoinositol phospholipid membrane anchor of human erythrocyte acetylcholinesterase by fast atom bombardment mass spectrometry. J. Biol. Chem. 263, 18776-18784 (1988).

41. Hirata, T. et al. Glycosylphosphatidylinositol mannosyltransferase II is the rate-limiting enzyme in glycosylphosphatidylinositol biosynthesis under limited dolichol-phosphate mannose availability. J. Biochem. 154, 257-264 (2013).

42. Naito, Y., Hino, K., Bono, H. \& Ui-Tei, K. CRISPRdirect: software for designing CRISPR/Cas guide RNA with reduced off-target sites. Bioinformatics 31, 1120-1123 (2015).

43. Ohba, C. et al. PIGN mutations cause congenital anomalies, developmental delay, hypotonia, epilepsy, and progressive cerebellar atrophy. Neurogenetics 15, 85-92 (2014).

44. Nakamura, N. et al. Expression cloning of PIG-L, a candidate Nacetylglucosaminyl-phosphatidylinositol deacetylase. J. Biol. Chem. 272, 15834-15840 (1997). 
45. Horie, K. et al. A homozygous mutant embryonic stem cell bank applicable for phenotype-driven genetic screening. Nat. Methods 8, 1071-1077 (2011).

46. $\mathrm{Xu}, \mathrm{X}$. et al. The genomic sequence of the Chinese hamster ovary (CHO)-K1 cell line. Nat. Biotechnol. 29, 735-741 (2011).

47. Choi, S. et al. New algorithm for the identification of intact disulfide linkages based on fragmentation characteristics in tandem mass spectra. J. Proteome Res. 9, 626-635 (2010).

48. Jones, D. T., Taylor, W. R. \& Thornton, J. M. A model recognition approach to the prediction of all-helical membrane protein structure and topology. Biochemistry 33, 3038-3049 (1994).

49. Sonnhammer, E. L., von Heijne, G. \& Krogh, A. A hidden Markov model for predicting transmembrane helices in protein sequences. Proc. Int. Conf. Intell. Syst. Mol. Biol. 6, 175-182 (1998).

50. Hofmann, K. \& Stoffel, W. TMbase - a database of membrane spanning proteins segments. Biol. Chem. Hoppe-Seyler 374, 166 (1993).

51. Tusnady, G. E. \& Simon, I. Principles governing amino acid composition of integral membrane proteins: application to topology prediction. J. Mol. Biol. 283, 489-506 (1998).

52. Kall, L., Krogh, A. \& Sonnhammer, E. L. Advantages of combined transmembrane topology and signal peptide prediction--the Phobius web server. Nucleic Acids Res. 35, W429-W432 (2007).

53. Altschul, S. F., Gish, W., Miller, W., Myers, E. W. \& Lipman, D. J. Basic local alignment search tool. J. Mol. Biol. 215, 403-410 (1990).

54. Berman, H. M. et al. The Protein Data Bank. Nucleic Acids Res. 28, 235-242 (2000).

55. Wu, S. \& Zhang, Y. LOMETS: a local meta-threading-server for protein structure prediction. Nucleic Acids Res 35, 3375-3382 (2007).

56. Lobley, A., Sadowski, M. I. \& Jones, D. T. pGenTHREADER and pDomTHREADER: new methods for improved protein fold recognition and superfamily discrimination. Bioinformatics 25, 1761-1767 (2009).

57. McGuffin, L. J., Bryson, K. \& Jones, D. T. The PSIPRED protein structure prediction server. Bioinformatics 16, 404-405 (2000).

58. Shen, M. Y. \& Sali, A. Statistical potential for assessment and prediction of protein structures. Protein Sci. 15, 2507-2524 (2006).

59. Melo, F., Sanchez, R. \& Sali, A. Statistical potentials for fold assessment. Protein Sci. 11, 430-448 (2002).

60. Vizcaino, J. A. et al. 2016 update of the PRIDE database and its related tools. Nucleic Acids Res. 44, D447-D456 (2016).

\section{Acknowledgements}

We thank Dr. J.F. Dubremetz (Montpellier University) for T5 mAb, Dr. Y. Tashima for discussion, K. Nakamura and Y. Kabumoto for cell sorting, and K. Kinoshita and A.

Kawate for technical help. T.H. was supported by grant-in-aid for Japan Society for the
Promotion of Science Fellows and for Research Activity start-up. This work was supported by JSPS/MEXT KAKENHI Grant Numbers JP16H04753 and JP17H06422 to T.K and a grant for Joint Research Project of the Research Institute for Microbial Diseases, Osaka University to Y.Y. and T.K. S.K.M. was supported by the Tokyo Biochemical Research Foundation.

\section{Author contributions}

T.H. performed experiments and analyzed the data. S.K.M. and Y.Y. created the 3D structure model. S.N. and D.M. conducted next-generation sequencing and analyzed the data. K.S. and Y.T. performed mass spectrometry and analyzed the data. M.F. and T.K. developed the research concept and M.F. initiated the research. N.K., Y.M., and Y.M. gave critical discussions. T.H., S.K.M., S.N., K.S., Y.Y., and T.K. wrote the manuscript. All authors contributed to editing the manuscript.

\section{Additional information}

Supplementary Information accompanies this paper at https://doi.org/10.1038/s41467017-02799-0.

Competing interests: The authors declare no competing financial interests.

Reprints and permission information is available online at http://npg.nature.com/ reprintsandpermissions/

Publisher's note: Springer Nature remains neutral with regard to jurisdictional claims in published maps and institutional affiliations.

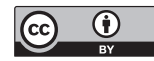

Open Access This article is licensed under a Creative Commons Attribution 4.0 International License, which permits use, sharing, adaptation, distribution and reproduction in any medium or format, as long as you give appropriate credit to the original author(s) and the source, provide a link to the Creative Commons license, and indicate if changes were made. The images or other third party material in this article are included in the article's Creative Commons license, unless indicated otherwise in a credit line to the material. If material is not included in the article's Creative Commons license and your intended use is not permitted by statutory regulation or exceeds the permitted use, you will need to obtain permission directly from the copyright holder. To view a copy of this license, visit http://creativecommons.org/ licenses/by/4.0/.

(C) The Author(s) 2018 